\title{
A Study on the Prediction of Aerofoil Trailing-edge Noise for Wind-turbine Applications
}

\author{
Alex Siu Hong Lau ${ }^{1}$, Jae Wook Kim ${ }^{1}$, Jeremy Hurault ${ }^{2}$ and Tomas Vronsky ${ }^{2}$ \\ ${ }^{1}$ Aerodynamics and Flight Mechanics Research Group, Aeronautics and Astronautics, University of Southampton, SO17 1BJ, UK \\ ${ }^{2}$ Vestas, Blade Technology Centre, West Medina Mills, Stag Lane, Newport, Isle of Wight, PO30 5TR, UK
}

\begin{abstract}
This paper presents a comparative study between BPM (Brooks, Pope and Marcolini) and TNO (TNO Institute of Applied Physics) models for the prediction of aerofoil trailing-edge noise with particular emphasis on wind-turbine applications. In this work, two enhanced versions of the BPM model are proposed and their performances are compared against two recent anisotropic TNO models that require more detailed boundary-layer information than the BPM-based models. The two current enhanced models are denoted as BPMM-PVII and BPMM-BL $k \omega$, where the former uses a panel method with viscous-inviscid interaction implemented and the latter employs a two-dimensional Reynolds-averaged Navier-Stokes model for boundary-layer calculations. By comparing the predicted sound spectra with existing measurement data for seven different aerofoils tested in the current study, it is shown that the BPMM-PVII model exhibits superior results to those by the other models for most cases despite the simplicity without considering anisotropy. The BPMM-PVII model is then combined with Prandtl's nonlinear lifting-line theory to calculate and investigate three-dimensional rotor noise characteristics of an NREL UAE Phase-VI wind turbine. It is demonstrated that the current approach may provide an efficient solution for the prediction of rotor aerodynamics and noise facilitating industrial design and development for low-noise wind turbines. Copyright (c) 2015 John Wiley \& Sons, Ltd.
\end{abstract}

\section{KEYWORDS}

Wind turbine; Aerofoil trailing-edge noise; Noise prediction model

\section{Correspondence}

Aerodynamics and Flight Mechanics Research Group, Aeronautics and Astronautics, University of Southampton, SO17 1BJ, UK.

E-mail: Alex.Lau@soton.ac.uk

Received ...

\section{INTRODUCTION}

An increasingly large portion of renewable energy has been produced by wind turbines in recent years. As the wind turbines grow in size and number, environmental noise becomes one of the major concerns for the general public [1,2]. There are many types of noise generation mechanisms associated with wind turbines, one of which is the aerodynamic noise generated by turbulent boundary layers scattered over the trailing-edge region of a wind-turbine blade. It is well known that trailing-edge (TE) noise is a major wind turbine noise source. An advancement in the capability of predicting/estimating TE noise will certainly contribute to the design and development of low-noise wind turbines. 
Although TE noise has been investigated extensively by many researchers using semi-empirical [3, 4, 5, 6], experimental [7, 8, 9] and advanced numerical [10,11, 12] methods, its efficient and accurate prediction in a reasonable time frame for practical design purposes remains a challenge for wind energy industry. For modern wind turbines, the Reynolds numbers $(\mathrm{Re})$ of the flow near the blade tips are in the order of several millions. It is therefore unrealistic to use CFD-based methods on a daily basis to calculate both turbulent boundary layers and the TE noise of a turbine blade, particularly for design optimisation purposes. On the other hand, the accuracy and flexibility of many semi-empirical noise prediction models, which are fast to return results, are still questionable. Hence there is an increasingly high demand to enhance the TE noise prediction capability for wind-energy industry.

Currently, there are two well-established prediction models being used widely in wind-energy industry: so-called BPM and TNO. The BPM model was developed by Brooks, Pope and Marcolini [3] based on measured aeroacoustic data from a NACA0012 aerofoil in a low-turbulence potential core of a free jet located in an anechoic chamber. The more recent TNO model was first proposed by Parchen [13] from the TNO Institute of Applied Physics in the Netherlands. Both methods are computationally fast and easy to implement. The BPM model uses curve fittings to be able to predict results outside its underlying measurement flow and geometric conditions, whereas the TNO model is more based on the physics of turbulent boundary layers demanding more data input. Some notable improvements in the TNO model have been suggested very recently, i.e. modelling the effects of flow anisotropy; and, the use of Reynolds-averaged Navier-Stokes (RANS) simulations $[14,15,16]$.

The present work aims to develop a reliable methodology to predict the TE noise emitted from an entire wind-turbine rotor (rather than just an aerofoil section). As it requires three-dimensional calculations, a fast semi-empirical prediction model is desired for practical purposes. Therefore, this paper begins with re-visiting and investigating the BPM model, from which an enhanced version is proposed after a comprehensive test and validation process (by using seven different aerofoils) compared against two latest anisotropic TNO models proposed by Bertagnolio et al. [15] and Kamruzzaman et al. [16]. For the estimations of the boundary-layer (BL) displacement thicknesses required by the BPM formulations, the current code is coupled with the BL prediction codes EDDYBL [17] and XFoil [18] for the utilisation of Wilcox $k-\omega$ 2D turbulence model and a panel method with viscous-inviscid interaction implemented (PVII) respectively. The enhanced BPM model is denoted as BPMM-PVII when only the PVII method is used, and BPMM-BLk $\omega$ when Wilcox $k-\omega$ turbulence model is employed. The boundary and initial conditions required by the Wilcox model is provided by a preliminary PVII calculation. The BPMM-PVII model is then incorporated with a three-dimensional rotor aerodynamics code based on Prandtl's nonlinear lifting-line theory in order to enable the noise prediction of an entire wind turbine.

The organization of the paper is as follows. In Section 2, the original BPM and the enhanced BPM models are explained. In Section 3, the anisotropic TNO models used in the current study are briefly recapitulated. In Section 4, comprehensive test cases are demonstrated for the validation of the two enhanced BPM models against the original BPM model, the TNO models and other experimental data. Then, the use of the BPMM-PVII model to estimate the noise from an entire wind-turbine rotor is presented in Section 5. Finally, some conclusions are drawn in Section 6.

\section{THE ENHANCED BPM MODEL}

\subsection{The Original BPM Model}

The original BPM model [3] makes the prediction of aerofoil TE noise in a 1/3-octave sound-pressure-level (SPL) spectrum based on the following formulae:

$$
\mathrm{SPL}_{\text {total }}=10 \log _{10}\left(10^{\mathrm{SPL}_{\alpha} / 10}+10^{\mathrm{SPL}_{s} / 10}+10^{\mathrm{SPL}_{p} / 10}\right)
$$


with

$$
\begin{aligned}
& \mathrm{SPL}_{p}=10 \log _{10}\left(\frac{\delta_{p}^{*} \mathrm{M}^{5} L \bar{D}_{h}}{r_{e}^{2}}\right)+A \frac{S t_{p}}{S t_{1}}+\left(K_{1}-3\right)+\Delta K_{1}, \\
& \mathrm{SPL}_{s}=10 \log _{10}\left(\frac{\delta_{s}^{*} \mathrm{M}^{5} L \bar{D}_{h}}{r_{e}^{2}}\right)+A \frac{S t_{s}}{S t_{1}}+\left(K_{1}-3\right), \\
& \mathrm{SPL}_{\alpha}=10 \log _{10}\left(\frac{\delta_{s}^{*} \mathrm{M}^{5} L \bar{D}_{h}}{r_{e}^{2}}\right)+B \frac{S t_{s}}{S t_{2}}+K_{2},
\end{aligned}
$$

where the subscripts " $p$ ", " $s$ " and " $\alpha$ " denote the pressure-side, the suction-side and the angle-of-attack, respectively; $\delta^{*}$ is the BL displacement thickness; $\mathrm{M}$ is the incident Mach number; $r_{e}$ is the retarded observer distance from the TE; $S t$ is the Strouhal number; $S t_{1}, S t_{2}, K_{1}, \Delta K_{1}$ and $K_{2}$ are either empirical functions or constants derived from the experimental data [3]; $A=A(\mathrm{Re})$ and $B=B(\mathrm{Re})$ are empirical shape functions for the 1/3-octave spectral shape and are functions of Re; $L$ is the aerofoil span; and $\bar{D}_{h}$ is the high-frequency directivity function for TE noise given by

$$
\bar{D}_{h}=\frac{2 \sin ^{2}\left(\Theta_{e} / 2\right) \sin ^{2} \Phi_{e}}{\left(1+\mathrm{M} \cos \Phi_{e}\right)\left[1+\left(\mathrm{M}-\mathrm{M}_{c}\right) \cos \Phi_{e}\right]},
$$

where $\mathbf{M}_{c}$ is the convection Mach number and is assumed to be $0.8 \mathrm{M} . \Theta_{e}$ and $\Phi_{e}$ are used to define the position of the observer from the centre of the TE as shown in Figure 1. The Strouhal numbers for the suction- and pressure-sides are given by $S t_{s}=f \delta_{s}^{*} / U_{\infty}$ and $S t_{p}=f \delta_{p}^{*} / U_{\infty}$ respectively, where $f$ is the frequency in $\mathrm{Hz}$ and $U_{\infty}$ is the incident freestream velocity. The original BPM model assumes that when $\alpha>12.5^{\circ}, \mathrm{SPL}_{\alpha}$ dominates and

$$
\begin{aligned}
& \operatorname{SPL}_{p}=-\infty \\
& \mathrm{SPL}_{s}=-\infty \\
& \mathrm{SPL}_{\alpha}=10 \log _{10}\left(\frac{\delta_{s}^{*} \mathrm{M}^{5} L \bar{D}_{l}}{r_{e}^{2}}\right)+A^{\prime} \frac{S t_{s}}{S t_{2}}+K_{2},
\end{aligned}
$$

where $\bar{D}_{l}$ is the low-frequency directivity function, and $A^{\prime}=A(3 \mathrm{Re})$.

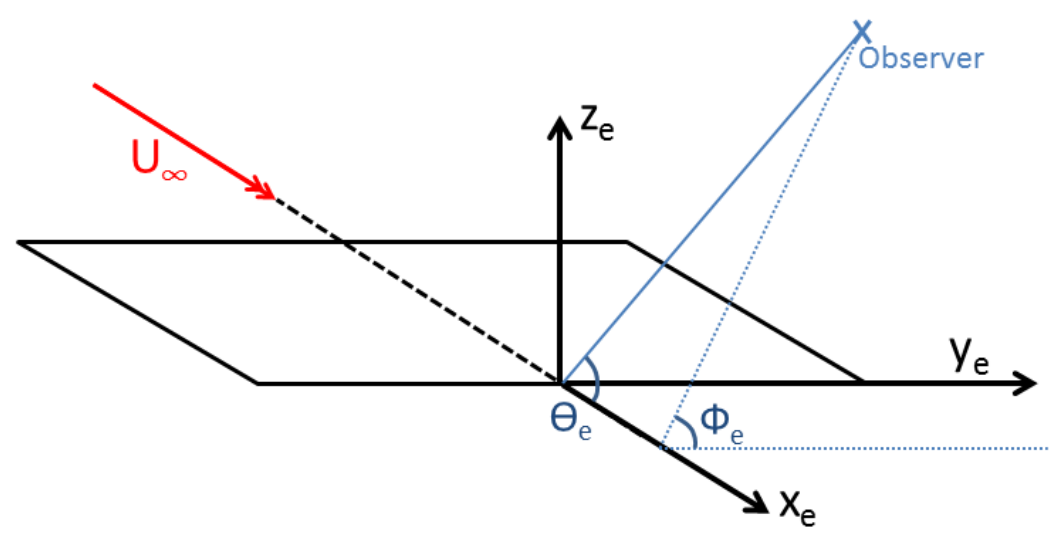

Figure 1. Observer position relative to the centre of the TE of an aerofoil section

The original BPM model was developed based on the measured aeroacoustic data solely from NACA0012 aerofoil. In this section, the original BPM model is applied to RisoB1-18 aerofoil and the results are compared with the corresponding wind-tunnel measurement data. For this task, the PVII method is used to calculate $\delta^{*}$ instead of using the empirical formula given in the original BPM model. The experimental measurements were conducted in the Laminar Wind Tunnel (LWT) of the Institute for Aerodynamics and Gasdynamics (IAG) of the University of Stuttgart by Vestas, Ltd. During the 
measurement, $U_{\infty}$ was kept at $70 \mathrm{~m} / \mathrm{s}$ and four values of $\alpha$ were studied: $3.4^{\circ}, 5.1^{\circ}, 7.1^{\circ}$ and $8.9^{\circ}$. Both tripped and clean $\mathrm{BL}$ conditions were tested. For the tripped condition, zigzag tapes with an opening angle of $60^{\circ}$, a thickness of $0.38 \mathrm{~mm}$ and a streamwise extent of $11 \mathrm{~mm}$ were used at $10 \%$ streamwise position to chord $(x / c)$ position on the pressure-side, and at $5 \% x / c$ on the suction-side. The chord length of the aerofoil model was $c=0.6 \mathrm{~m}$. The propagated sound was measured at an observer position $1 \mathrm{~m}$ directly above the mid-span of the TE. The coherent particle velocity (CPV) method [19, 20], which is based on a cross-spectral analysis of two hot-wire sensor signals placed on the suction- and the pressure-sides of the aerofoil TE, was used to measure the TE noise. The low frequency $(<1000 \mathrm{~Hz})$ measurements by the CPV method are known to be disturbed by hydrodynamic fluctuations [20], hence they can only be used as rough indications of the spectral trend. The resulting normalised 1/3-octave SPL spectra for the tripped and clean conditions are shown in Figure 2. For each BL condition, the maximum measured SPL value among all the cases with different $\alpha$ is used to normalise the measured and the predicted SPL spectra. It can be seen that the spectral shapes of the BPM prediction and the measurement are similar for frequencies higher than approximately $1000 \mathrm{~Hz}$. However, it was observed that the BPM model over-predicted the SPL by approximately $5 \mathrm{~dB}$ in the high-frequency range.
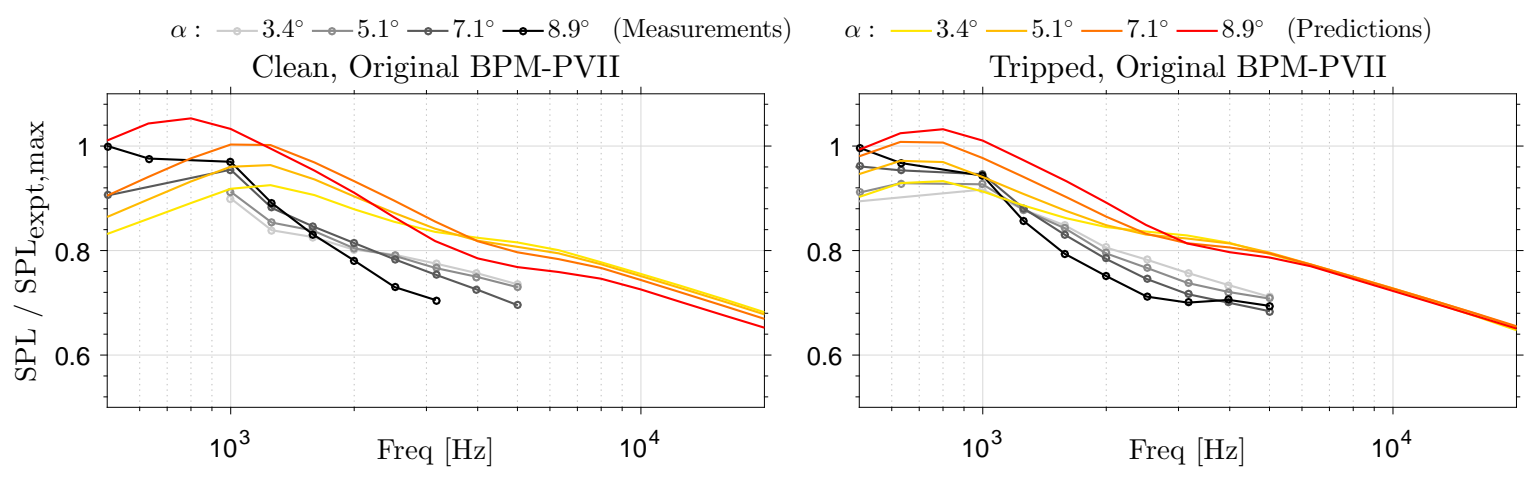

Figure 2. RisoB1-18 aerofoil normalised 1/3-octave SPL spectra

\subsection{The Current Modifications}

The current study uses the wind-tunnel-measured SPL spectra of several modern aerofoils used on wind turbines to derive an enhanced BPM model. The measured spectra need to be scaled for this analysis. Fink [21], when scaling airframe noise data where TE noise was believed to be dominant, assumed a universal spectrum shape $F(S t)$ for the noise. This normalisation assumed a fifth-power relation to the incident velocity. The current scaling procedure follows that used by Brooks, Pope and Marcolini [3], which was in line to the fifth-power relationship suggested by Fink. For an aerofoil at non-zero $\alpha$, the measured $1 / 3$-octave SPL spectra are scaled according to

$$
\mathrm{SPL}_{\text {scaled }}=\mathrm{SPL}-10 \log _{10}\left(\mathrm{M}^{5} \frac{\delta_{s}^{*} L}{r_{e}^{2}}\right),
$$

and the suction-side Strouhal number $S t_{s}$ is used as the dimensionless frequency. From a scaled spectrum, the peak scaled SPL and the Strouhal number at which it occurs, denoted by $S t_{\text {peak }}$, can be found.

The first two empirical functions considered are $S t_{1}$ and $S t_{2}$, which relate $S t_{\text {peak }}$ to the incident $\mathrm{M}$ and $\alpha$. Brooks, Pope and Marcolini [3] found that $S t_{\text {peak }}$ showed no clear dependence on Re, and suggested

$$
S t_{1}=0.02 \mathrm{M}^{-0.6}
$$




$$
S t_{2}=S t_{1} \times\left\{\begin{array}{cll}
1 & \text { for } & \alpha<1.33^{\circ} ; \\
10^{0.0054(\alpha-1.33)^{2}} & \text { for } & 1.33^{\circ} \leqslant \alpha \leqslant 12.5^{\circ} \\
4.72 & \text { for } & 12.5^{\circ}<\alpha,
\end{array}\right.
$$

For the current enhanced model, it is modified to

$$
\begin{gathered}
S t_{1, \text { new }}=0.022 \mathrm{M}^{-0.6}, \\
S t_{2, \text { new }}=S t_{1, \text { new }} \times\left\{\begin{array}{cll}
1 & \text { for } & \alpha<1.33^{\circ} ; \\
10^{0.003(\alpha-1.33)^{2}} & \text { for } & 1.33^{\circ} \leqslant \alpha \leqslant 14^{\circ} ; \\
4.72 & \text { for } & 14^{\circ}<\alpha,
\end{array}\right.
\end{gathered}
$$

It is worth noting that the limiting $\alpha$ of $12.5^{\circ}$ in Eq. 9 would give rise to very different sound spectral shape for $\alpha$ higher than approximately $8^{\circ}$ to $9^{\circ}$. Hence, the limiting $\alpha$ for the enhanced model is increased to $14^{\circ}$ as indicated in Eq. 11 . The remaining two empirical coefficients to modify are $K_{1}$ and $K_{2}$. They relate the scaled SPL to Re and $\alpha$. The constant $K_{1}$ is the peak scaled SPL value for zero $\alpha$. Originally, for $\operatorname{Re}>8.0 \times 10^{5}, K_{1}=128.5$. It can be seen from Figure 2 that the original BPM model over-predicts the SPL, particularly for the clean condition. Hence, it is proposed to reduce the value of $K_{1}$ as follows:

$$
K_{1, \text { new }}=\left\{\begin{array}{cc}
128.5\left(1-\sigma_{\text {clean }}\right) & \text { for a clean condition, } \\
128.5\left(1-\sigma_{\text {tripped }}\right) & \text { for a tripped condition, }
\end{array}\right.
$$

where the reduction factors $\sigma_{\text {clean }}$ and $\sigma_{\text {tripped }}$ are both less than $2 \%$ (under confidentiality) in this work, which could be further improved by including additional datasets later on. The function $K_{2}$ relates the peak scaled SPL and $\alpha$. It is related to $K_{1}$ and is also a function of M. In the original BPM model, it is given by

$$
K_{2}=K_{1}+\left\{\begin{array}{cll}
-1000 & \text { for } & \alpha<\gamma_{0}-\gamma \\
\sqrt{\beta^{2}-(\beta / \gamma)^{2}\left(\alpha-\gamma_{0}\right)^{2}}+\beta_{0} & \text { for } & \gamma_{0}-\gamma \leqslant \alpha \leqslant \gamma_{0}+\gamma \\
-12 & \text { for } & \gamma_{0}+\gamma<\alpha,
\end{array}\right.
$$

with

$$
\begin{aligned}
& \gamma=27.094 \mathrm{M}+3.31, \quad \gamma_{0}=23.43 \mathrm{M}+4.651, \\
& \beta=72.65 \mathrm{M}+10.74, \quad \beta_{0}=-34.19 \mathrm{M}-13.82 .
\end{aligned}
$$

For the enhanced model, $\beta$ and $\beta_{0}$ are modified as follows:

$$
\beta_{\text {new }}=72.65 \mathrm{M}+3, \quad \beta_{0, \text { new }}=-34.19 \mathrm{M}-6.7 .
$$

The remaining modification focuses on the prediction of the BL displacement thickness $\delta^{*}$. The current code is coupled with the BL solvers XFoil and EDDYBL for the utilisation of a panel method with viscous-inviscid interaction implemented (PVII) and the Wilcox $k-\omega$ 2D turbulence model respectively. The current BPMM-PVII model uses only the PVII method for the $\delta^{*}$ calculations. The PVII-predicted suction-side $\delta^{*}$ at the TE, denoted by $\delta_{\mathrm{SS}}^{*}$, have been compared to the corresponding measured values in the Second Benchmark-problem for Airframe Noise Computations (BANC-II) workshop [22] cases 1 to 4 . A NACA0012 aerofoil with $0.4 \mathrm{~m}$ chord was used for these four cases. The results of the comparison are shown in Table I. It can be seen that PVII estimates smaller $\delta_{\mathrm{SS}}^{*}$ than the experimental measurements for all four cases. Some preliminary studies, which are not presented in the current paper, were conducted to check the effect of increasing the PVII-estimated $\delta_{\mathrm{SS}}^{*}$ on the BPM-predicted SPL spectrum. It was found that increasing $\delta_{\mathrm{SS}}^{*}$ leads to small increase in SPL for low frequencies and small reduction in SPL at higher frequencies. This reduction in SPL becomes very small at even higher frequencies. For different aerofoils under various conditions, the bounding frequencies 
and the changes in SPL of these differently-affected regions are different. It will be shown in Section 4 that increasing the PVII-predicted $\delta_{\mathrm{SS}}^{*}$ by $10 \%$, together with the modified parameters of the enhanced BPM model, lead to better agreement to experimental SPL measurements for many aerofoils under different conditions compared to the original BPM model. Therefore, the PVII-predicted $\delta_{\mathrm{SS}}^{*}$ is increased by $10 \%$ for the current BPMM-PVII model.

\begin{tabular}{|c|c|c|c|c|c|c|}
\hline Case & $\operatorname{Re} / 10^{6}$ & $\mathrm{M}$ & $\alpha\left[^{\circ}\right]$ & PVII $\delta_{\text {SS }}^{*}[\mathrm{~mm}]$ & Expt. $\delta_{\text {SS }}^{*}[\mathrm{~mm}]$ & $\%$ diff. to Expt. \\
\hline \hline 1 & 1.5 & 0.1664 & 0 & 2.53 & 2.97 & -14.65 \\
\hline 2 & 1.5 & 0.1641 & 4 & 3.62 & 4.76 & -24.05 \\
\hline 3 & 1.5 & 0.1597 & 6 & 4.43 & 5.67 & -21.93 \\
\hline 4 & 1.0 & 0.1118 & 0 & 2.73 & 3.14 & -13.13 \\
\hline
\end{tabular}

Table I. PVII-predicted and experimentally-measured displacement thicknesses for the NACA0012 aerofoils used in the BANC-II workshop

The current BPMM-BLk $\omega$ model uses Wilcox $k-\omega$ 2D turbulence model for BL calculations. The initial and boundary conditions required by the model is provided by a preliminary PVII calculation. The $k$ - $\omega$-estimated $\delta_{\mathrm{SS}}^{*}$ is used directly without any modification.

\section{THE TNO MODEL}

The TNO TE noise model does not rely entirely on empirical relationships, and takes more turbulent flow properties near the TE into account. For a turbulent boundary layer over a smooth, stationary and rigid surface at low Mach number, the wavenumber frequency spectrum of the surface pressure fluctuations is given by

$$
\Phi_{p}\left(k_{1}, k_{3}, \omega\right)=4 \rho_{0}^{2}\left[k_{1}^{2} /\left(k_{1}^{2}+k_{3}^{2}\right)\right] \int_{0}^{\infty}\left\{\Lambda_{2}\left\langle u_{2}^{2}\right\rangle\left(\partial U_{1} / \partial y_{2}\right)^{2} \Phi_{22} \Phi_{m} e^{-2|\boldsymbol{k}| y_{2}}\right\} d y_{2}
$$

where the subscripts " 1 ", " 2 " and " 3 " denote the streamwise, wall-normal and spanwise directions respectively; $y_{2}$ is the wall-normal distance; $\omega$ is the angular frequency; $k_{1}, k_{2}$ and $k_{3}$ are the elements of the wavenumber vector; $\Lambda_{2}=\Lambda_{2}\left(y_{2}\right)$ is the wall-normal correlation length; $\left\langle u_{2}^{2}\right\rangle$ is the wall-normal Reynolds stress component; $\partial U_{1} / \partial y_{2}$ is the wall-normal gradient of the local streamwise mean velocity; $\Phi_{22}=\Phi_{22}\left(k_{1}, k_{3}, k_{e}\right)$ is the normalised turbulence spectral tensor diagonal component (or spectrum) associated to the wall-normal velocity fluctuation after being integrated over $k_{2}$, with $k_{e}$ being the wavenumber of the energy-containing eddies; and $\Phi_{m}=\Phi_{m}\left(\omega-U_{c}\left(y_{2}\right) k_{1}\right)$ is the moving-axis spectrum, which describes how the turbulent velocity spectrum is distorted by the evolution of eddies as they convect past the TE.

By comparing the asymptotic behaviour of the von Karman spectrum and the Kolmogorov spectrum for the inertial subrange for isotropic turbulence [14, 23], $k_{e}$ is given by

$$
k_{e} \approx\left(\frac{1.5 \times 27 \sqrt{\pi} \Gamma(1 / 3)}{110 \Gamma(5 / 6)}\right)^{3 / 2} \frac{\epsilon}{k_{T}^{3 / 2}} \approx 1.90 \frac{\epsilon}{k_{T}^{3 / 2}},
$$

where $\Gamma$ is the gamma function, $\epsilon$ is the mean energy dissipation rate and $k_{T}$ is the turbulent kinetic energy. $\Phi_{m}$ has a Gaussian form

$$
\Phi_{m}=\frac{1}{\alpha_{2} \sqrt{\pi}} \exp \left[-\left(\frac{\omega-U_{c} k_{1}}{\alpha_{2}}\right)^{2}\right],
$$

where $U_{c}$ is the convective velocity and is approximated by $U_{c}=0.7 U_{1}$. For a frequency range in which the aerofoil can be considered non-compact, i.e. the sound wavelength is smaller than the chord length, the far-field pressure spectrum can be expressed as

$$
S(\omega)=\left.\frac{L}{4 \pi r_{e}^{2}} \int_{-\infty}^{\infty} \frac{\omega}{c_{0} k_{1}} \Phi_{p}\left(k_{1}, \omega\right)\right|_{k_{3}=0} d k_{1},
$$


where $c_{0}$ is the speed of sound.

Eqs. 16 to 19 are commonly used by the two TNO models considered. The BL properties required are estimated using the Wilcox $k-\omega$ model included in EDDYBL. Note that all the spectra presented in this paper are one-sided but the spectrum described by Eq. 19 is double-sided. Hence a factor of two is needed to be multiplied to Eq. 19.

\subsection{Bertagnolio Anisotropic TNO Model}

By introducing three anisotropy stretch factors $\beta_{1}, \beta_{2}$, and $\beta_{3}$, which stretch the isotropic von Karman spectrum in the streamwise, wall-normal and spanwise directions respectively, Bertagnolio et al. [15] derived anisotropic expressions for $\Phi_{22}$ and $\Lambda_{2}$. The three stretch factors are given by

$$
\begin{aligned}
& \beta_{1}=0.4 ; \\
& \beta_{2}=\gamma^{1 / 5} \\
& \beta_{3}=(2 \gamma)^{1 / 2}
\end{aligned}
$$

where $\gamma$ is the non-dimensional mean pressure gradient and is defined as

$$
\gamma=\frac{\delta}{u_{\tau}}\left[\frac{\left(\partial P / \partial y_{1}\right)^{2}}{\rho_{0} \mu}\right]^{1 / 3}
$$

where $P$ is the mean surface pressure, $u_{\tau}$ is the friction velocity, $\delta$ is the BL thickness and $\mu$ is the dynamic viscosity. $\Phi_{22}$ then becomes dependent on $\beta_{1}$ and $\beta_{3}$, such that

$$
\Phi_{22}=\frac{4}{9 \pi}\left(\frac{1}{k_{e}}\right)^{2} \beta_{1} \beta_{3} \frac{\left(\beta_{1} k_{1} / k_{e}\right)^{2}+\left(\beta_{3} k_{3} / k_{e}\right)^{2}}{\left[1+\left(\beta_{1} k_{1} / k_{e}\right)^{2}+\left(\beta_{3} k_{3} / k_{e}\right)^{2}\right]^{7 / 3}} .
$$

$\Lambda_{2}$ becomes dependent on $\beta_{1}$ and $\beta_{2}$, i.e.

$$
\Lambda_{2}=\frac{55 \Gamma(1 / 3)}{108 \sqrt{\pi} \Gamma(17 / 6)} \frac{1}{k_{e}} \beta_{2} \frac{3+11\left(\beta_{1} k_{c} / k_{e}\right)^{2}}{3+8\left(\beta_{1} k_{c} / k_{e}\right)^{2}} \frac{1}{\sqrt{1+\left(\beta_{1} k_{c} / k_{e}\right)^{2}}}
$$

where $k_{c}=\omega / U_{c}$ is the convective wavenumber. $\left\langle u_{2}^{2}\right\rangle$ is given by assuming isotropic turbulence, i.e.

$$
\left\langle u_{2}^{2}\right\rangle=\frac{2}{3} k_{T}
$$

This anisotropic TNO model by Bertagnolio et al. is referred to as FB-Aniso TNO herein.

\subsection{Kamruzzaman Anisotropic TNO Model}

Kamruzzaman et al. [16] suggested a correction for $\epsilon$ near the wall, such that

$$
\epsilon_{\mathrm{mod}}=\left[c_{5} \frac{c_{3}}{c_{1}}\left(y^{+}\right)^{b\left(c_{2}-c_{4}\right)}\right] \epsilon
$$

where $\epsilon_{\text {mod }}$ is the modified mean energy dissipation rate, $c_{1}=4.2, c_{2}=1.1, c_{3}=0.03, c_{4}=0.8, c_{5}=15$ and $b=0.91$. This formula reduces $\epsilon$ near the wall.

The anisotropic corrections suggested by Kamruzzaman et al. [16] are based on the derivation of a semi-empirical anisotropy correction factor $f_{22}$. This factor is a function of the Reynolds number based on Taylor's microscale $\operatorname{Re}_{\lambda}$, 
which can be found by

$$
\begin{aligned}
& \lambda_{f}=\sqrt{15 \nu \frac{2}{3} \frac{k_{T}}{\epsilon_{\mathrm{mod}}}} ; \\
& \lambda_{g}=\lambda_{f} / \sqrt{2} \\
& \sigma=\sqrt{\frac{2}{3} k_{T}} \\
& \operatorname{Re}_{\lambda}=\sigma \lambda_{g} / \nu
\end{aligned}
$$

where $\nu$ is the kinematic viscosity. Then $f_{22}=\operatorname{Re}_{\lambda}{ }^{-0.09}$. The anisotropic $\left\langle u_{2}^{2}\right\rangle$ is then estimated as

$$
\left\langle u_{2}^{2}\right\rangle=\frac{2}{3} k_{T} f_{22}
$$

An anisotropic form of $\Lambda_{2}$ is used, such that

$$
\Lambda_{2} \approx 0.75 \frac{\left[\left\langle u_{2}^{2}\right\rangle\right]^{3 / 2}}{\epsilon} \approx 0.40 \frac{\left[k_{T} f_{22}\right]^{3 / 2}}{\epsilon}
$$

The anisotropy correction factor for a length scale is then given by $f_{L}=\left(f_{22}\right)^{3 / 2}$. Based on this, an anisotropic form of $\Phi_{22}$ is derived, such that

$$
\Phi_{22}=\frac{4}{9 \pi}\left(\frac{1}{k_{e}}\right)^{2} f_{L}^{2} \frac{\left(f_{L} k_{1} / k_{e}\right)^{2}+\left(f_{L} k_{3} / k_{e}\right)^{2}}{\left[1+\left(f_{L} k_{1} / k_{e}\right)^{2}+\left(f_{L} k_{3} / k_{e}\right)^{2}\right]^{7 / 3}},
$$

which is identical to Eq. 23 of the FB-Aniso TNO model if $f_{L}=\beta_{1}=\beta_{2}=\beta_{3}$. This anisotropic TNO model by Kamruzzaman et al. is referred to as MK-Aniso TNO herein.

\section{COMPARISONS OF THE MODEL PREDICTIONS AND EXPERIMENTAL MEASUREMENTS}

\begin{tabular}{|c|c|c|c|c|c|c|c|c|}
\hline Case group & Aerofoil & $c[\mathrm{~m}]$ & $L[\mathrm{~m}]$ & $U_{\infty}[\mathrm{m} / \mathrm{s}]$ & $\left.\alpha{ }^{\circ}\right]$ & BL Trip & $r_{e}[\mathrm{~m}]$ & Fig. \\
\hline \hline 1.1 & NACA64 $_{3}-418$ & 0.6 & 1.0 & 70.0 & 0.0 & No & 1.0 & 3 \\
\hline 1.2 & NACA64 $_{3}-418$ & 0.6 & 1.0 & 70.0 & $0.0,3.0$ & Yes & 1.0 & 4 \\
\hline \hline 2.1 & NACA0012 & 0.4 & 1.0 & 56.0 & 0.0 & Yes & 1.0 & 5 \\
\hline 2.2 & NACA0012 & 0.4 & 1.0 & 54.8 & 4.0 & Yes & 1.0 & 5 \\
\hline \hline 3.1 & DU96-180 & 0.3 & 1.0 & 60.0 & 4.0 & Yes & 1.0 & 6 \\
\hline \hline 4.1 & RisoB1-15 & 0.6 & 1.0 & 70.0 & $3.0,6.0,9.0,11.0$ & No & 1.0 & 7 \\
\hline 4.2 & RisoB1-15 & 0.6 & 1.0 & 70.0 & $3.0,6.0,9.0,11.0$ & Yes & 1.0 & 8 \\
\hline \hline 5.1 & RisoB1-18 & 0.6 & 1.0 & 70.0 & $3.4,5.1,7.1,8.9$ & No & 1.0 & 9 \\
\hline 5.2 & RisoB1-18 & 0.6 & 1.0 & 70.0 & $3.4,5.1,7.1,8.9$ & Yes & 1.0 & 10 \\
\hline \hline 6.1 & Aerofoil-18 & 0.6 & 1.0 & 70.0 & $2.0,4.0,6.0,9.0$ & No & 1.0 & 11 \\
\hline \hline 6.2 & Aerofoil-18 & 0.6 & 1.0 & 70.0 & $2.0,4.0,6.0,9.0$ & Yes & 1.0 & 12 \\
\hline 7.1 & Aerofoil-21 & 0.6 & 1.0 & 70.0 & $2.7,4.6,6.5,8.9$ & No & 1.0 & 13 \\
\hline 7.2 & Aerofoil-21 & 0.6 & 1.0 & 70.0 & $2.7,4.6,6.5,8.9$ & Yes & 1.0 & 14 \\
\hline
\end{tabular}

Table II. The test cases

The 38 test cases considered in the current paper are listed in Table II. Seven aerofoils are employed in the analysis. Aerofoil-18 and Aerofoil-21 have thickness to chord ratios of $18 \%$ and $21 \%$ respectively, and are used in modern large wind turbines. The data for case groups 1.1 and 1.2 are from Ref. [24], while those for case groups 2.1, 2.2 and 3.1 are from the BANC-II workshop [22] cases 1, 2 and 5 respectively. For case groups 4.1 to 7.2, the data were measured by Vestas in 


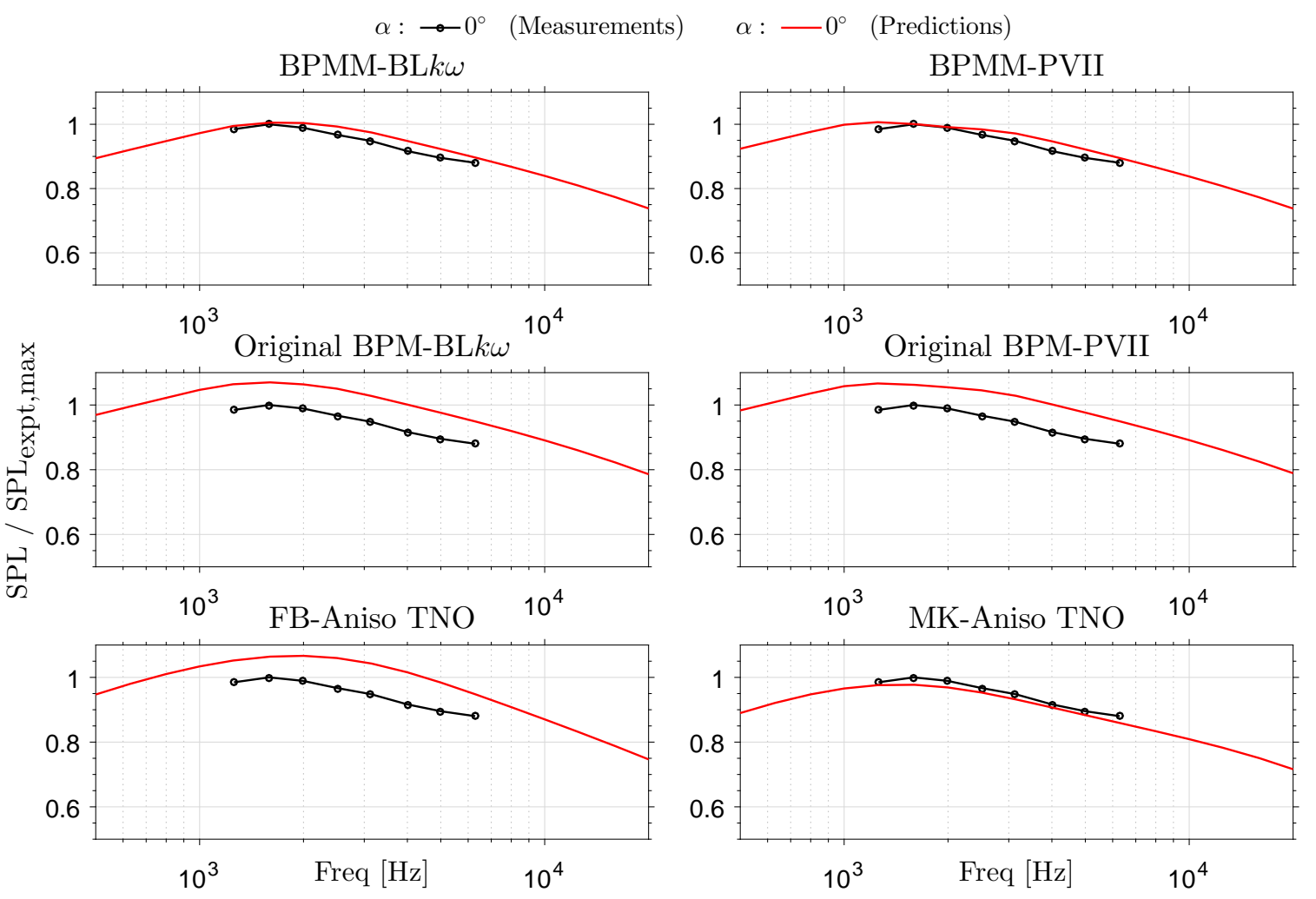

Figure 3. Test case 1.1 for a NACA64 $4_{3}-418$ aerofoil at $\alpha=0^{\circ}$ with clean condition (PVII denotes the panel method with viscousinviscid interaction implemented, BL $k \omega$ refers to Wilcox $k-\omega 2 \mathrm{D}$ turbulence model)

the LWT with the same experimental set-up as that described in section 2.1. Note that the current experimental data for the NACA0012, NACA64 3 -418 and DU-96-180 aerofoils are not used in the derivation of the enhanced BPM formulations.

For each case group, the maximum measured SPL value among all its test cases is used to normalise the measured and the predicted SPL spectra. The measured and the predicted normalised 1/3-octave SPL spectra by the enhanced BPM models, the original BPM model and the two anisotropic TNO models are presented in Figures 3 to 14. Note that the PVII and Wilcox $k-\omega$ methods have been used in conjunction with the original BPM model. So six sets of model predictions are presented in each of these figures. It can be seen from Figures 3 to 14 that in general, both enhanced BPM models are able to predict SPL spectra that show better agreement with experimental measurements than the other four noise models. In terms of both spectral shape and magnitude, the spectra predicted by both enhanced models, especially BPMM-PVII, show good agreement for most of the aerofoils analysed. Furthermore, Figures 3 to 6 show that even though the experimental data for the current NACA0012, NACA64 3 -418 and DU-96-180 aerofoils have not been used in the derivation of the enhanced BPM formulations, both enhanced models are able to predict the SPL spectra of these aerofoils well. From the measured SPL, it can be seen that in general, increasing $\alpha$ leads to relatively higher low-frequency noise and lower high-frequency noise. This trend can also be observed from the predicted SPL by the six noise models. In terms of the prediction of the frequency beyond which SPL becomes lower when $\alpha$ is increased for an aerofoil, Bertagnolio anisotropic TNO model shows the best performance. Note that due to the different boundary-layer prediction methods used in the current and other published studies, discrepancies for the TNO-predicted SPL spectra for the BANC-II workshop cases shown here and in other papers are possible. The TNO model predictions are presented to show the effects of using these two TNO formulations compared to the current BPMM-BL $k \omega$ model, which employs the same BL prediction method as these two TNO models. 


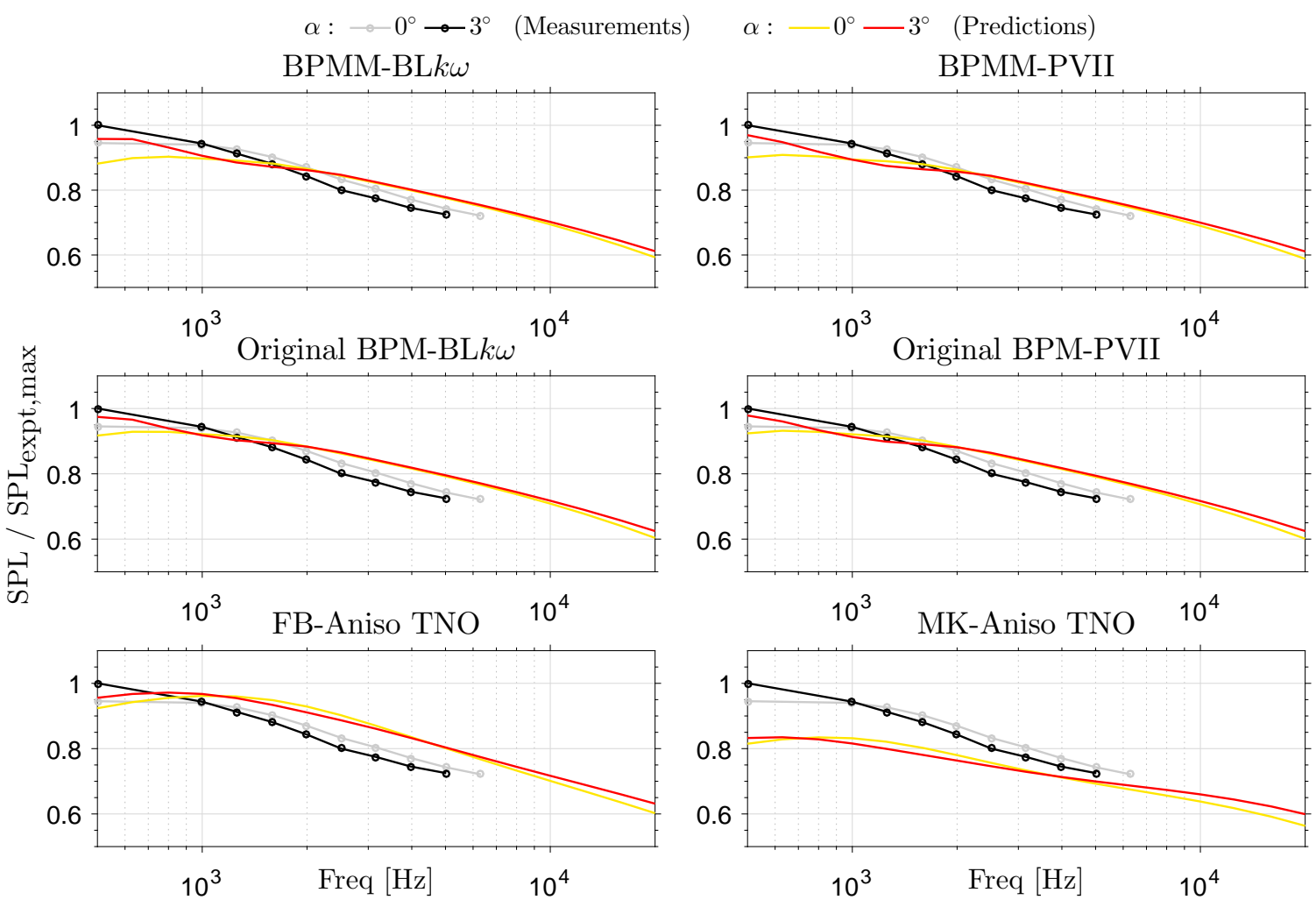

Figure 4. Test case 1.2 for a NACA64 $4_{3}-418$ aerofoil with tripped condition

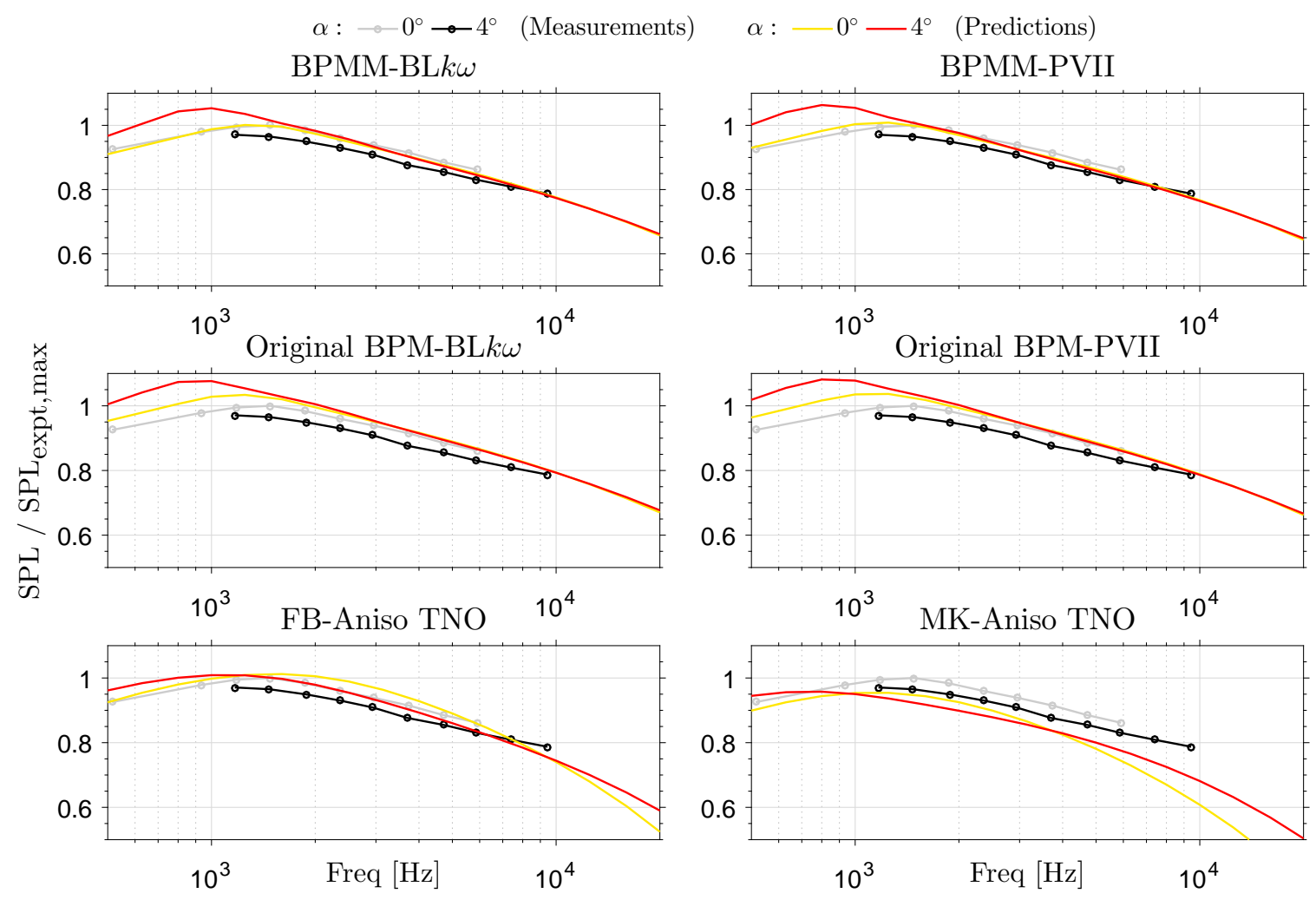

Figure 5. Test cases $2.1\left(\alpha=0^{\circ}\right)$ and $2.2\left(\alpha=4^{\circ}\right)$ for a NACA0012 aerofoil with $\mathrm{U}_{\infty}=56 \mathrm{~m} / \mathrm{s}$ and $54.8 \mathrm{~m} / \mathrm{s}$ respectively and tripped condition 


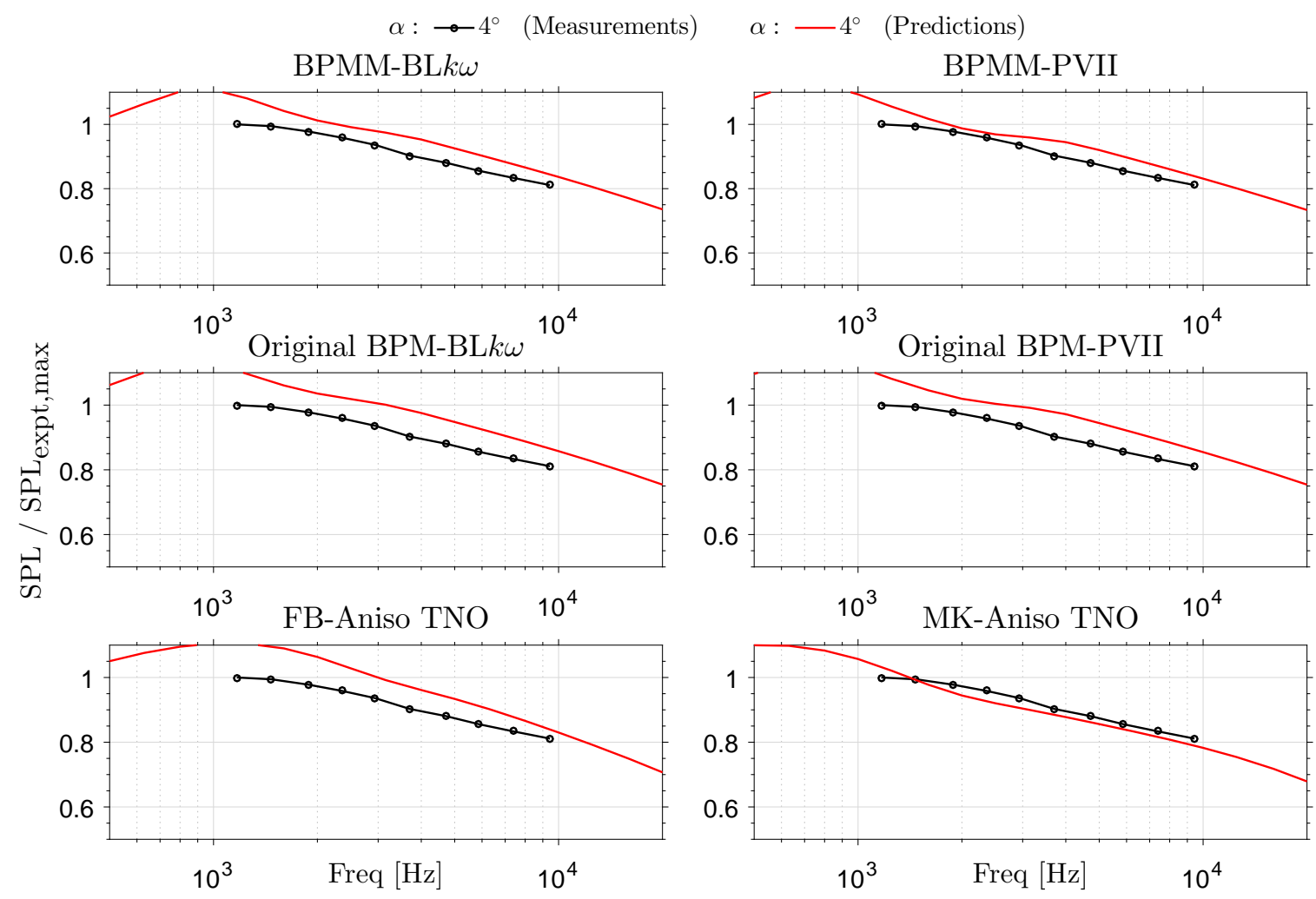

Figure 6. Test case 3.1 for a DU-96-180 aerofoil at $\alpha=4^{\circ}$ with tripped condition

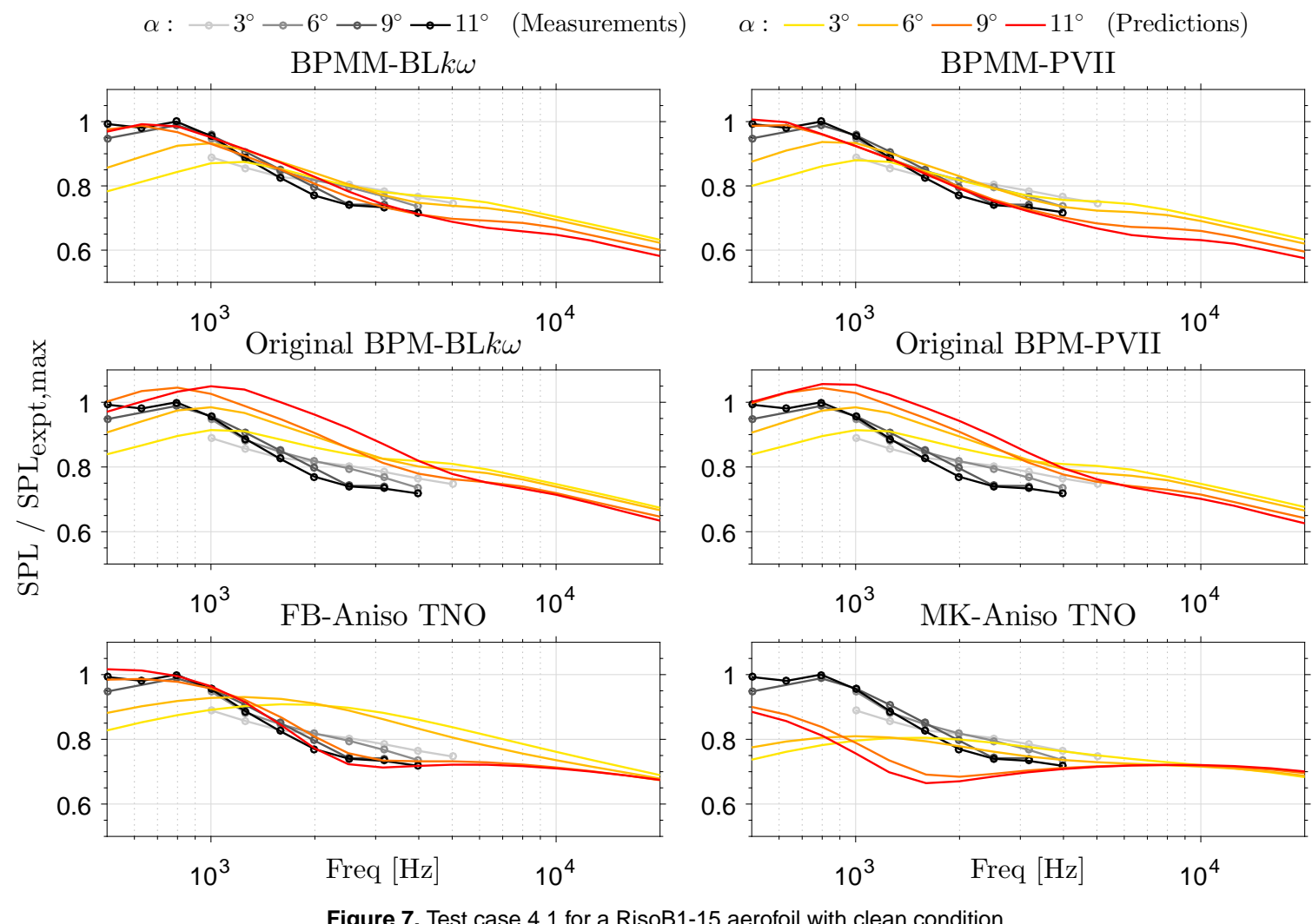

Figure 7. Test case 4.1 for a RisoB1-15 aerofoil with clean condition 


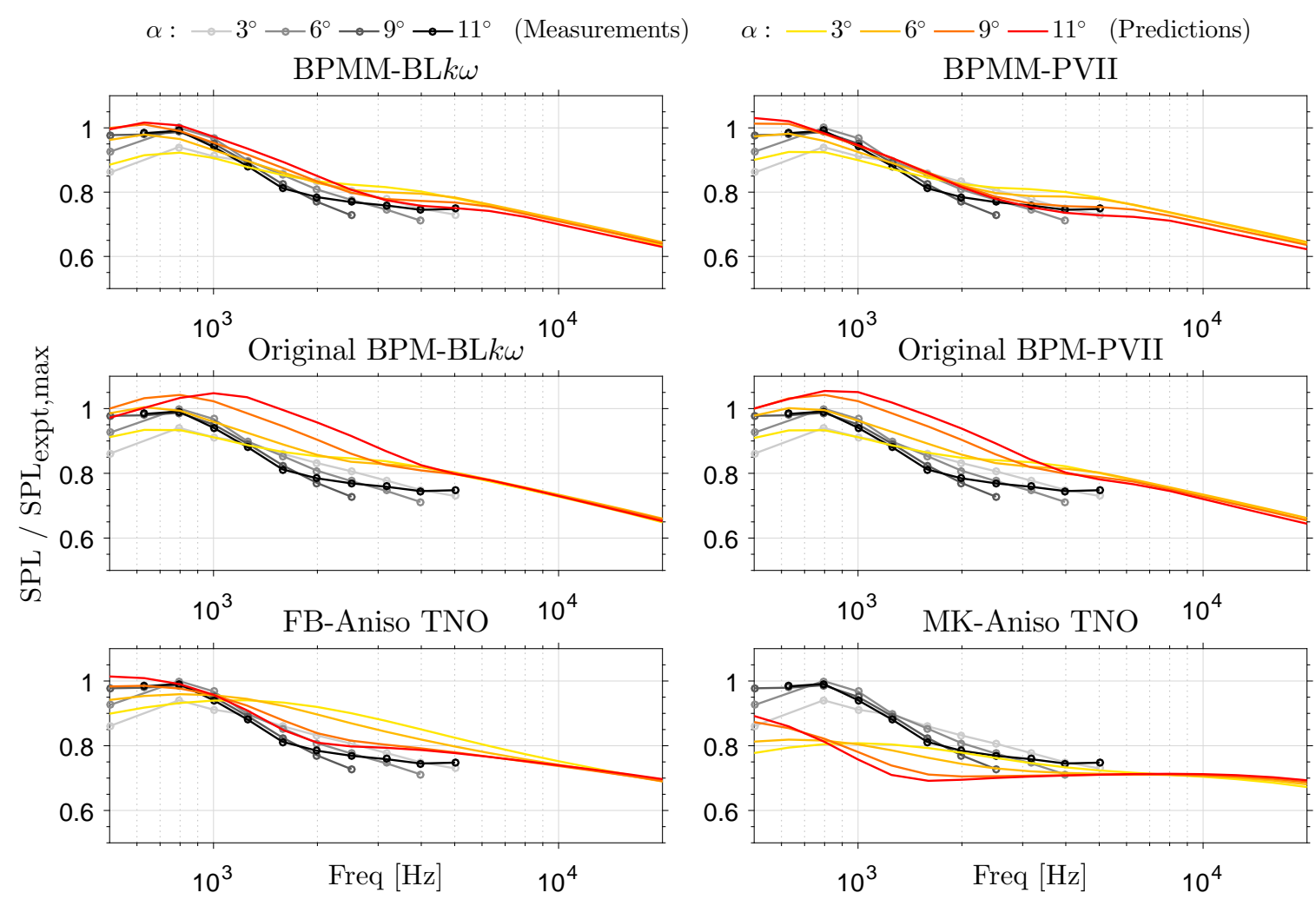

Figure 8. Test case 4.2 for a RisoB1-15 aerofoil with tripped condition

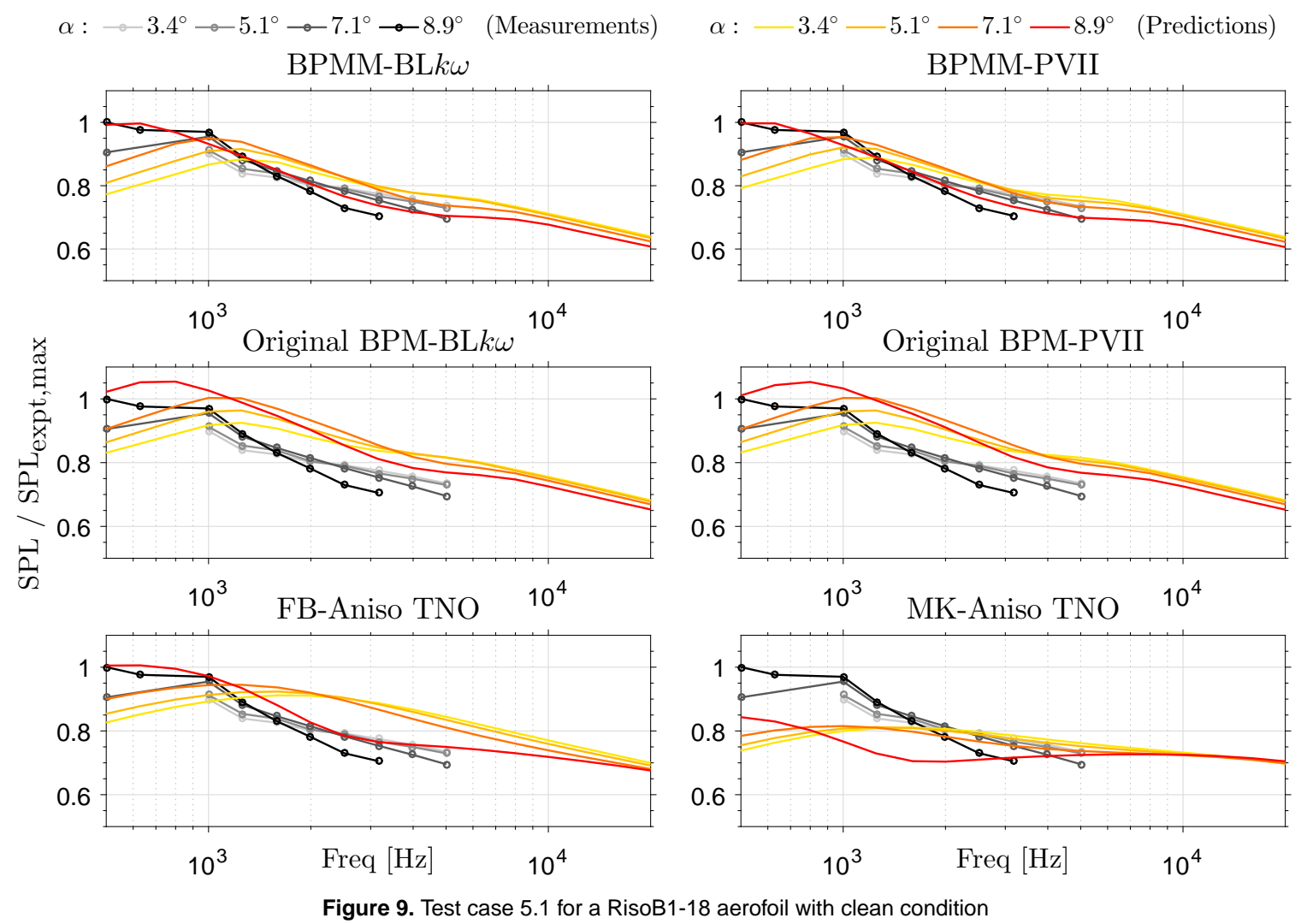


$\alpha: \multimap 3.4^{\circ} \multimap 5.1^{\circ} \rightarrow 7.1^{\circ} \rightarrow 8.9^{\circ} \quad$ (Measurements)

BPMM-BL $k \omega$
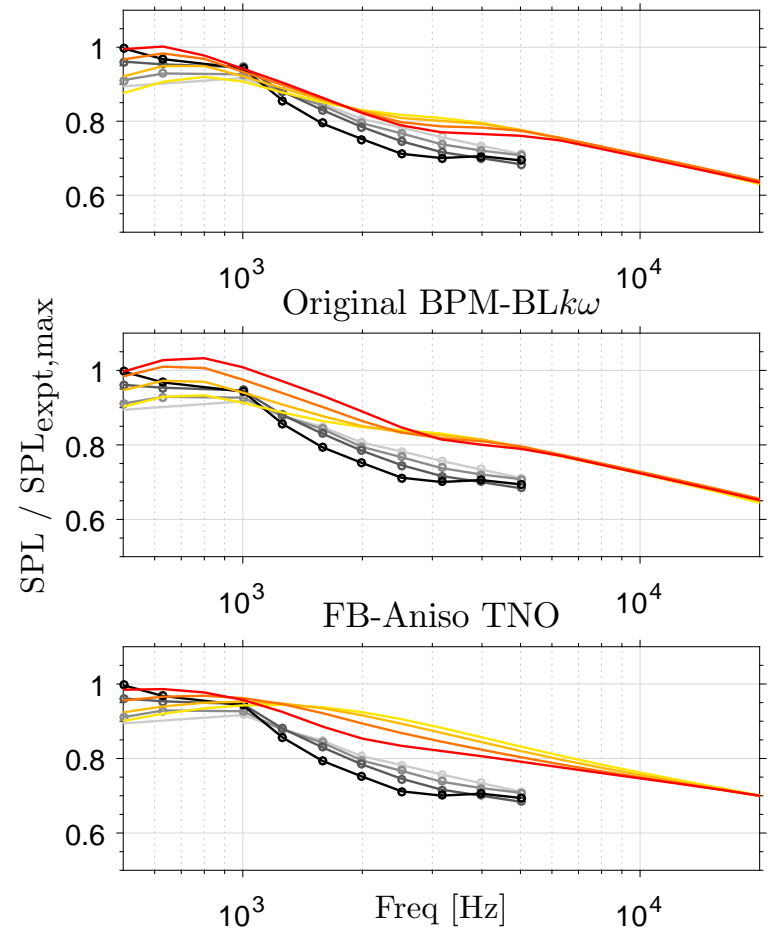

$\alpha:-3.4^{\circ}-5.1^{\circ}-7.1^{\circ}-8.9^{\circ} \quad$ (Predictions)

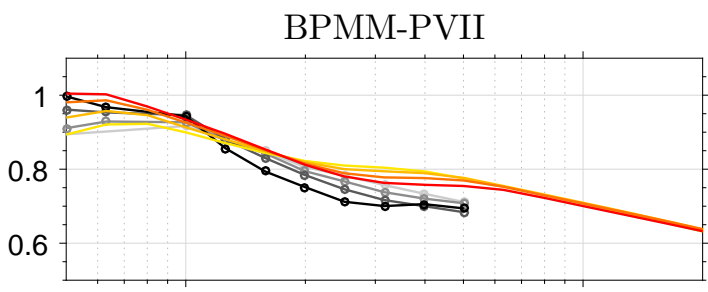

$10^{3}$ Original BPM-PVII ${ }^{10^{4}}$
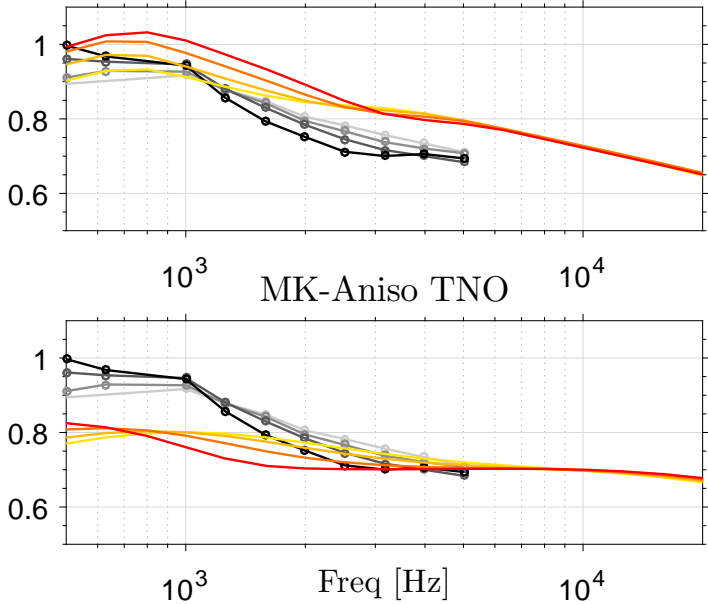

Figure 10. Test case 5.2 for a RisoB1-18 aerofoil with tripped condition

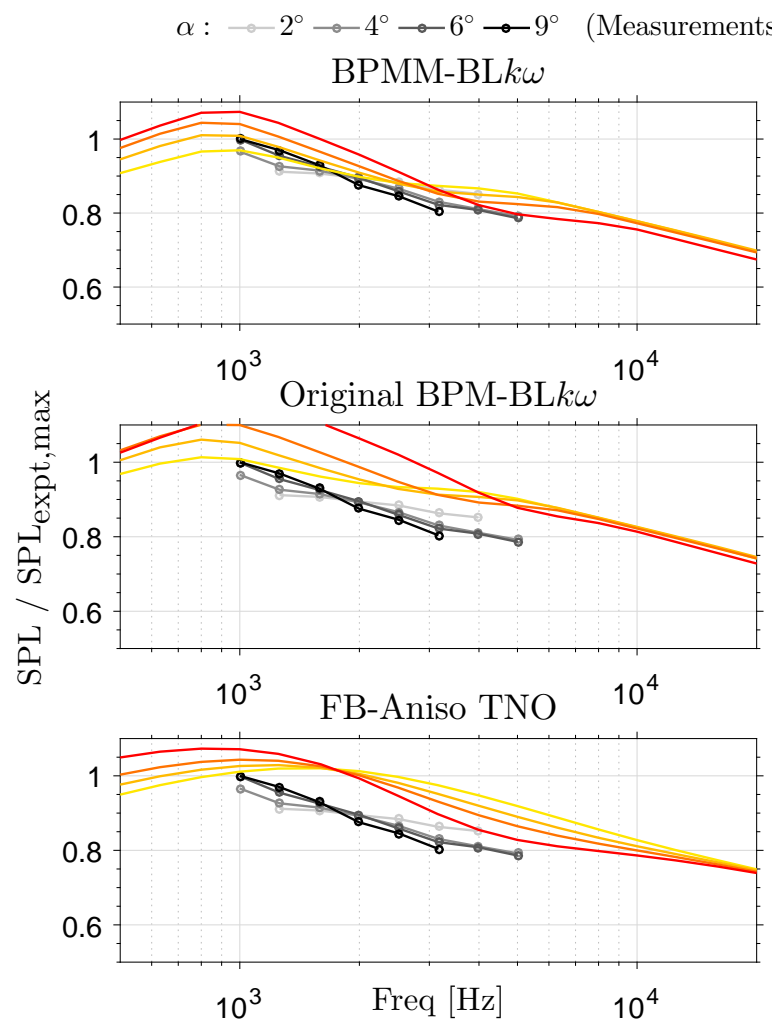

$\alpha:-2^{\circ}-4^{\circ}-6^{\circ}-9^{\circ} \quad$ (Predictions)

BPMM-PVII

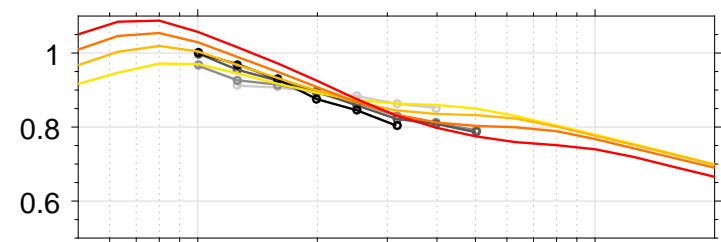

$10^{3}$ Original BPM-PVII ${ }^{10^{4}}$
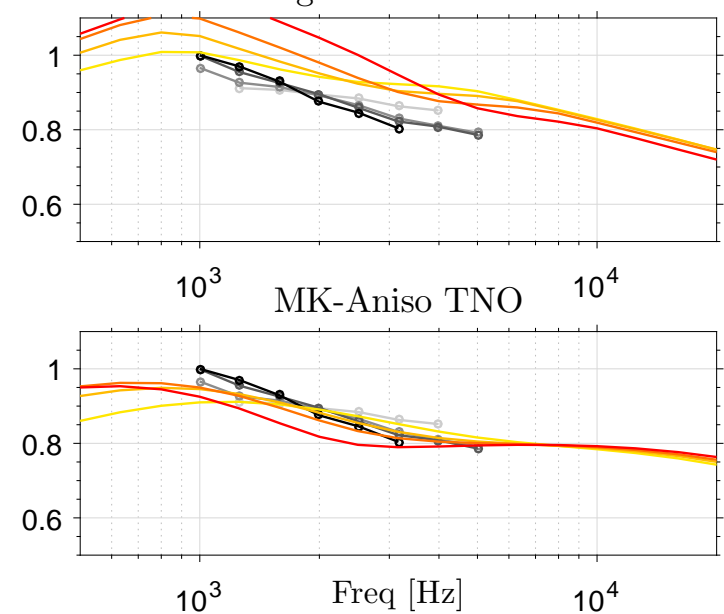

Figure 11. Test case 6.1 for Aerofoil-18 with clean condition 


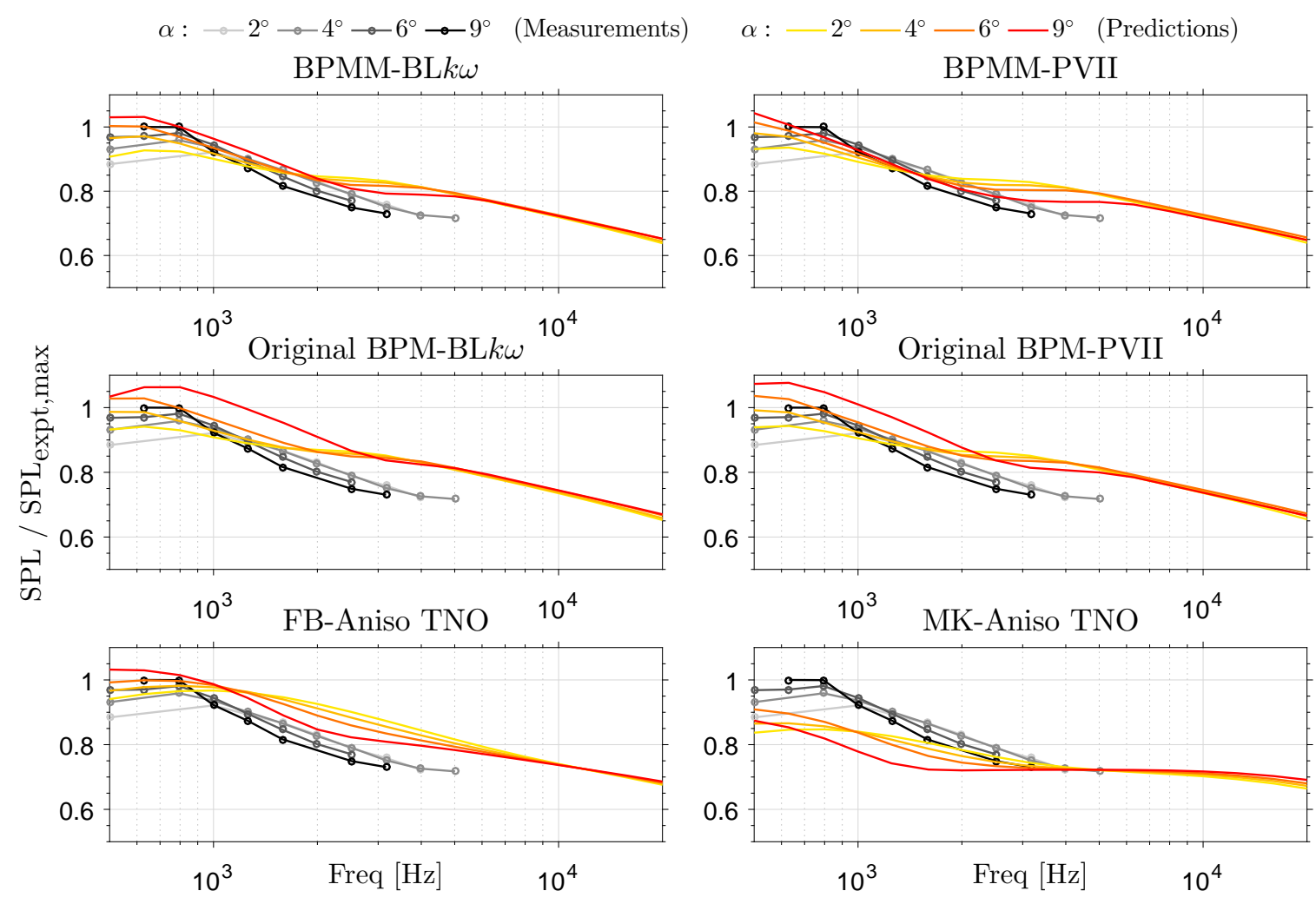

Figure 12. Test case 6.2 for Aerofoil-18 with tripped condition
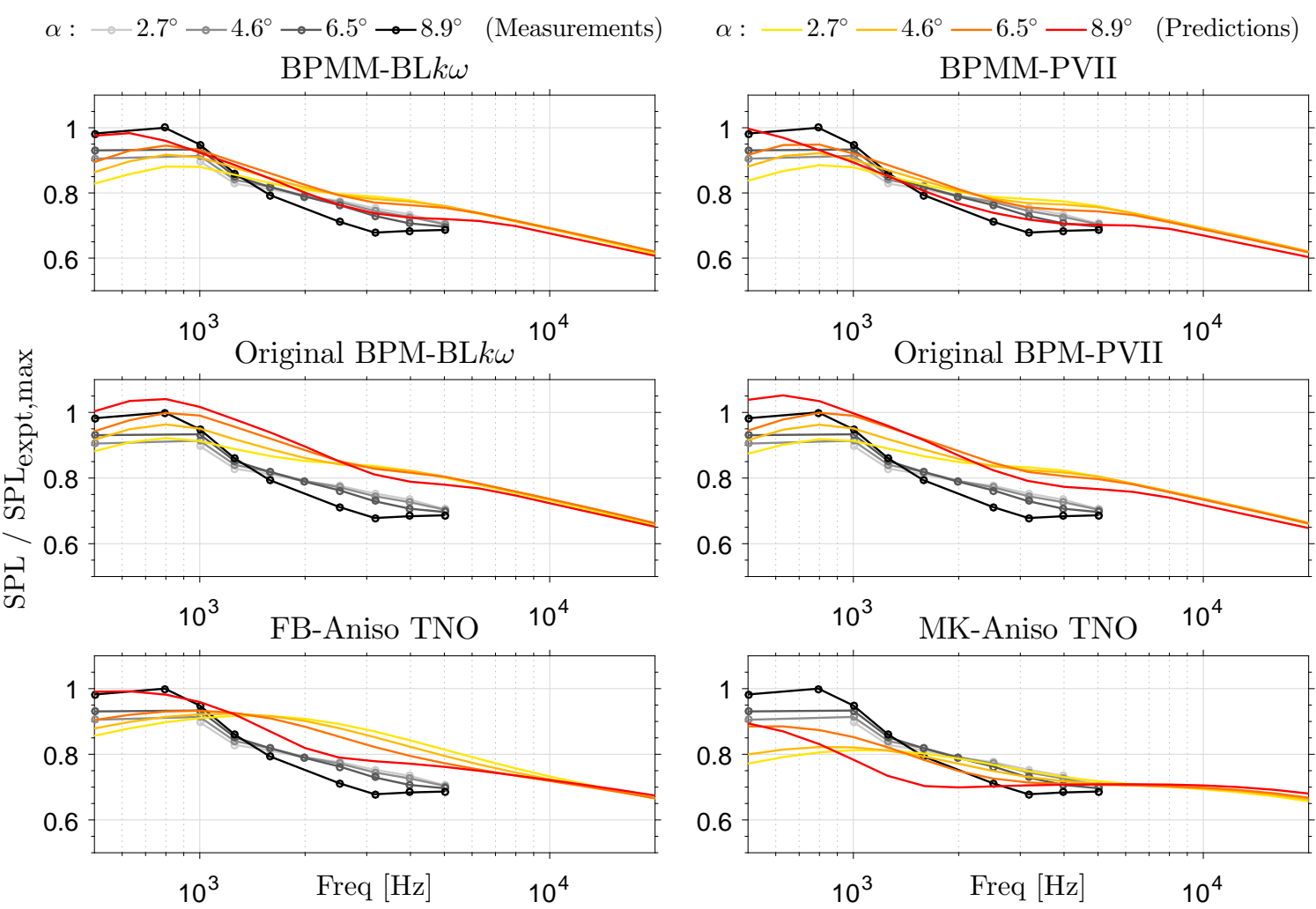

Figure 13. Test case 7.1 for Aerofoil-21 with clean condition 
$\alpha: \multimap 2.7^{\circ} \multimap 4.6^{\circ} \multimap 6.5^{\circ} \multimap 8.9^{\circ}$ (Measurements)

$\mathrm{BPMM}-\mathrm{BL} k \omega$

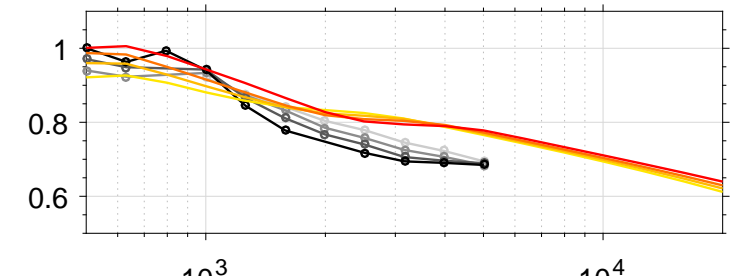

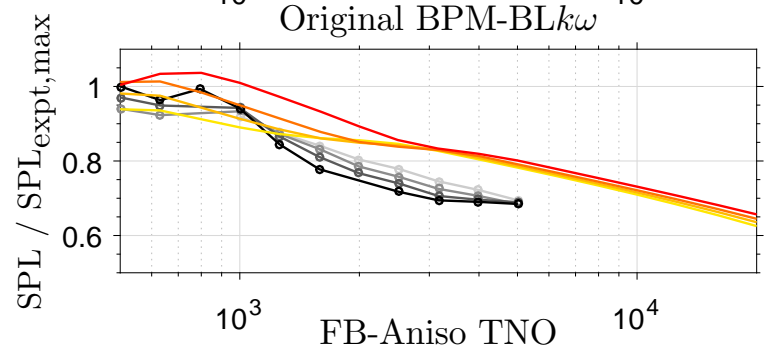

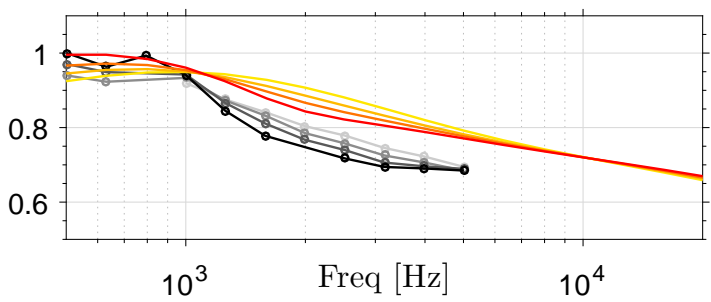

$\alpha:-2.7^{\circ}-4.6^{\circ}-6.5^{\circ}-8.9^{\circ}$ (Predictions)

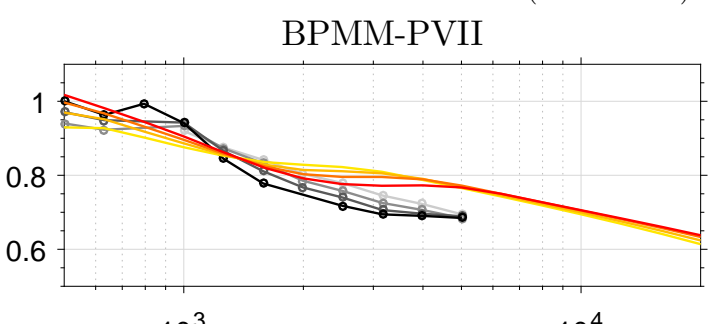

$10^{3}$

Original BPM-PVII ${ }^{10^{4}}$
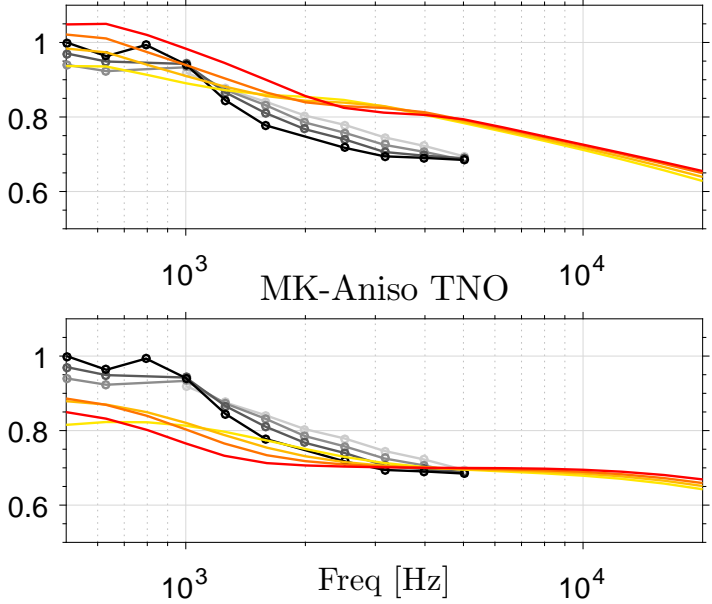

Figure 14. Test case 7.2 for Aerofoil-21 with tripped condition

To help quantifying the degree of agreement, a $L^{2}$-norm difference $\Delta$ of each model-predicted SPL spectrum to the corresponding experimental measurement is defined, such that

$$
\Delta_{k}=\sqrt{\frac{1}{f_{b}-f_{a}} \int_{f_{a}}^{f_{b}}\left(\frac{\text { SPL }_{\text {expt }}-\text { SPL }_{\text {model }}}{\text { SPLexpt }}\right)^{2} d f}
$$

where $k$ denotes one of the 38 test cases; $f_{a}$ and $f_{b}$ are the logarithmic centre-frequencies of the lower and upper frequency bands respectively; SPLexpt and SPL model are the experimentally-measured and the model-predicted SPL respectively. Note that the integration is with respect to the logarithm of the frequency, and $f_{a}$ and $f_{b}$ are determined by the availability of the experimental data for each case. Frequency bands lower than $1000 \mathrm{~Hz}$ are not considered due to the limits of the CPV experimental method as explained in section 2.1. For each of the six sets of model predictions, a mean $\Delta$ is defined such that

$$
\Delta_{\text {mean }}=\left(\frac{1}{38} \sum_{k=1}^{38} \Delta_{k}\right) .
$$

Figure 15 shows the value of $\Delta_{\text {mean }}$ and the standard deviation of $\Delta$ about $\Delta_{\text {mean }}$ for each of the six models. It can be seen that the $\Delta_{\text {mean }}$ and the standard deviation values of the BPMM-PVII model predictions are the smallest. The BPMM-BL $k \omega$ model leads to the second smallest $\Delta_{\text {mean }}$ and standard deviation values, which are smaller than those of the MK-Aniso TNO model. Therefore, the SPL spectra predicted by the BPMM-PVII model are the closest to the experimental measurements, followed by the BPMM-BL $k \omega$ model predictions. From this analysis and the previous qualitative comparisons of the predicted and the measured SPL spectra, it can be seen that both enhanced BPM models outperform the two anisotropic TNO models tested in terms of the closeness of the predicted SPL spectra to the measurements. 


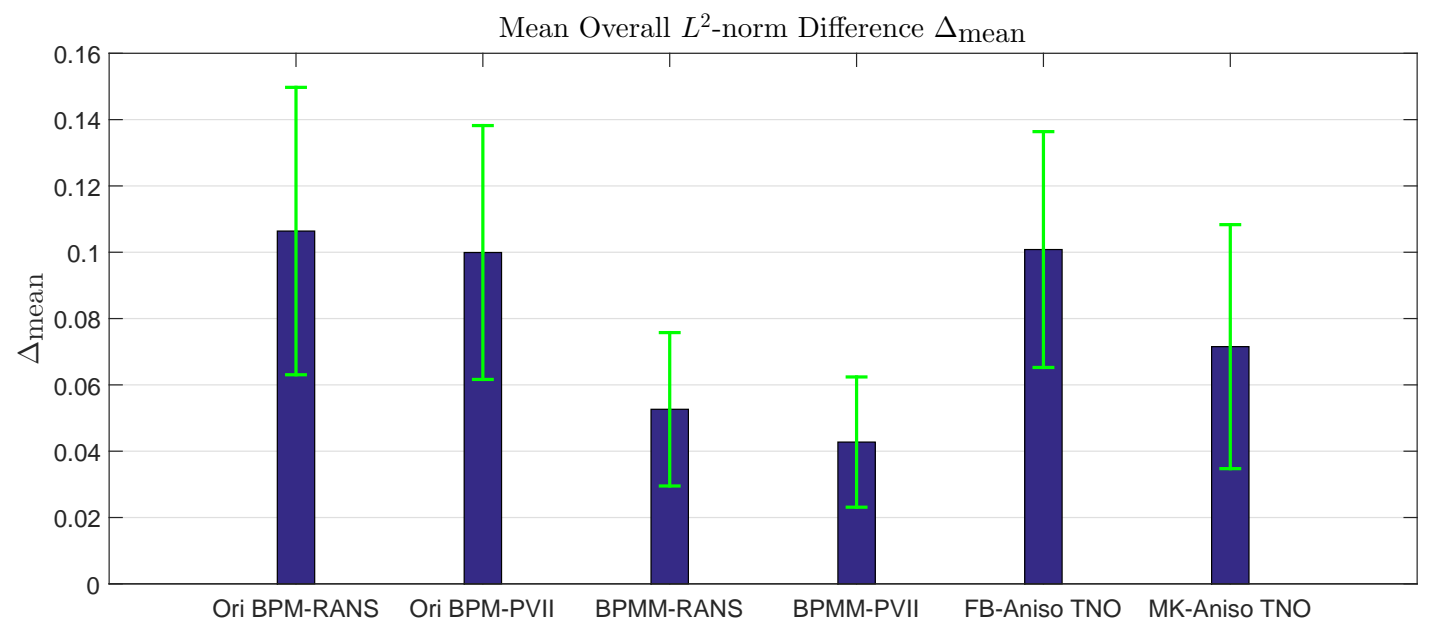

Figure 15. The mean overall $L^{2}$-norm difference $\Delta_{\text {mean }}$ between each set of model predictions and the measurements

\section{THE TRAILING-EDGE NOISE FROM A WIND TURBINE ROTOR}

This section demonstrates an exemplar of predicting TE noise in 3D space for an entire wind-turbine rotor based on the proposed BPMM-PVII model. The model wind turbine considered here is the NREL (National Renewable Energy Laboratory) UAE (Unsteady Aerodynamic Experiment) Phase-VI research wind turbine [25]. It was a two-bladed stallregulated wind turbine with a rotor diameter of $10.058 \mathrm{~m}$, a hub height of $12.192 \mathrm{~m}$, zero tilt and a rotational speed of 71.63 $\mathrm{rpm}$ (the velocity due to rotation alone $U_{\text {rot }}=37.7 \mathrm{~m} / \mathrm{s}$ at the blade tips). The blade cross-section changes from a circle to a S809 aerofoil within the first $16.5 \%$ of the span from the root, and the S809 is maintained outboard. Furthermore, the blades are tapered and twisted. Figure 16 shows the planform of the blade and the S809 aerofoil. The current study only considers an upwind configuration with zero cone angle, zero yaw and an uniform incident freestream velocity. With these simplifications, the aerodynamic states of a blade are independent of the blade azimuth. Figure 17 shows the global rotor coordinate system and the definition of the local blade coordinate denoted by " $y$ ".

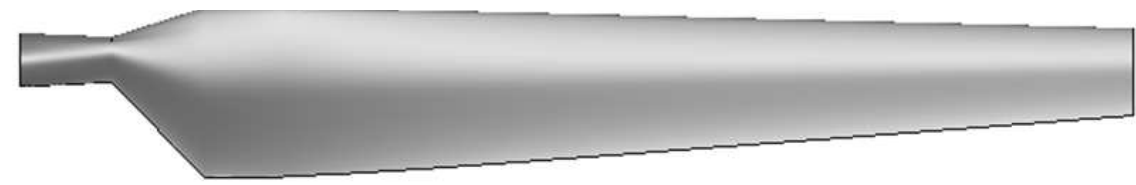

UAE Phase VI Blade Planform

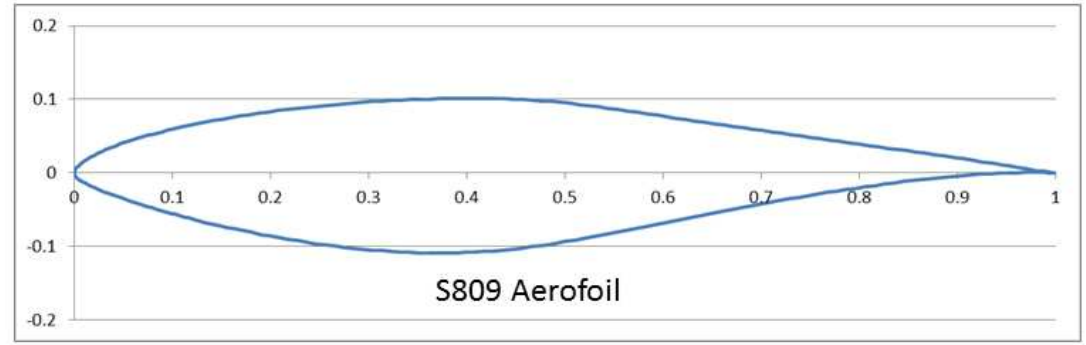

Figure 16. NREL UAE Phase VI wind turbine blade planform and the S809 aerofoil

In the current approach, the aerodynamic properties of the rotating blades are calculated by Prandtl's nonlinear liftingline theory. The current code assumed a helical wake shed from each blade. The radius of the helical wake is assumed 


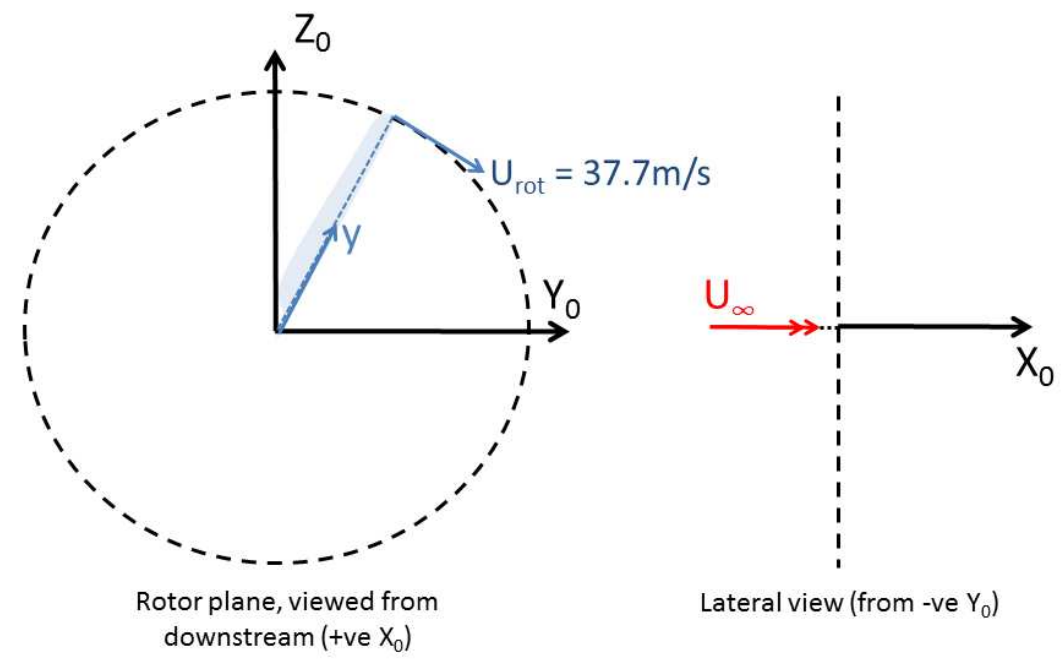

Figure 17. Global coordinate system and the definition of the local blade coordinate $y$

constant in the streamwise direction downstream of the rotor. Each blade is modelled by a straight lifting line which consists of $\mathrm{N}$ control points, where the aerodynamic properties are estimated. Each control point is half-way between its adjacent helical wake filaments. So for $\mathrm{N}$ control points on a blade, there are $\mathrm{N}$ horseshoe vortex systems and $\mathrm{N}+1$ helical wake filaments, which are discretised into many straight-line elements. The assumed helical wake geometry is shown in Figure 18. The velocity induced by all the elements of the helical wake filaments shed from all blades on each control point is estimated using the Biot Savart Law. For a helical wake with constant unit circulation, the velocity induced by an element of a helical wake filament on control point $m$ is given by

$$
u_{\text {element }}(m)=\frac{1}{4 \pi} \frac{\left(r_{1}+r_{2}\right)\left(\overrightarrow{r_{1}} \times \overrightarrow{r_{2}}\right)}{r_{1} r_{2}\left(r_{1} r_{2}+\overrightarrow{r_{1}} \cdot \overrightarrow{r_{2}}+\theta^{2} l^{2}\right)},
$$

where $r_{1}$ and $r_{2}$ are the distances between the two element end-points (denoted by 1 and 2 respectively) and the control point, $\overrightarrow{r_{1}}$ and $\overrightarrow{r_{2}}$ are the distance vectors from the element end-points to the control point, $l$ is the length of the wake element and $\theta=0.1$. The $\theta^{2} l^{2}$ term is added to avoid singularity as separation tends to zero. Then the wake elemental contributions are summed for all filaments, including that from the other blade. The induced velocity at control point $m$ is given by

$$
u_{j}(m)=\sum_{i}^{N} \Gamma_{i}\left[\bar{u}_{j}(m, i+1)-\bar{u}_{j}(m, i)\right],
$$

where the subscript $j=1,2$ and 3 represents the global streamwise, vertical and lateral directions respectively, $\Gamma_{i}$ is the circulation of the $i^{t h}$ horseshoe vortex system, and $\bar{u}_{j}(m, i)$ is a velocity component induced at control point $m$ by the $i^{t h}$ unit-strength helical vortex filament.

An iterative loop is started by first setting the induced velocity to zero. Then the initial angle of attack is simply

$$
\alpha_{i}=\arctan \left[U_{\infty} /\left(y_{i} \omega\right)\right]-\alpha_{g}-\alpha_{p}
$$

where $y_{i}$ is the radial location of the $i^{\text {th }}$ control point along the blade, $\omega=7.50 \mathrm{rad} / \mathrm{s}$ is the blade angular velocity, $\alpha_{g}$ and $\alpha_{p}$ are the geometric twist angle and the blade pitch angle respectively. From this initial angle of attack, the lift coefficient $C_{l, i}$ at each control point can be estimated. For the current calculation, the experimentally-measured lift curves for the S809 aerofoil from tests taken at the Colorado State University and the Ohio State University as stated in the 2003 NREL 


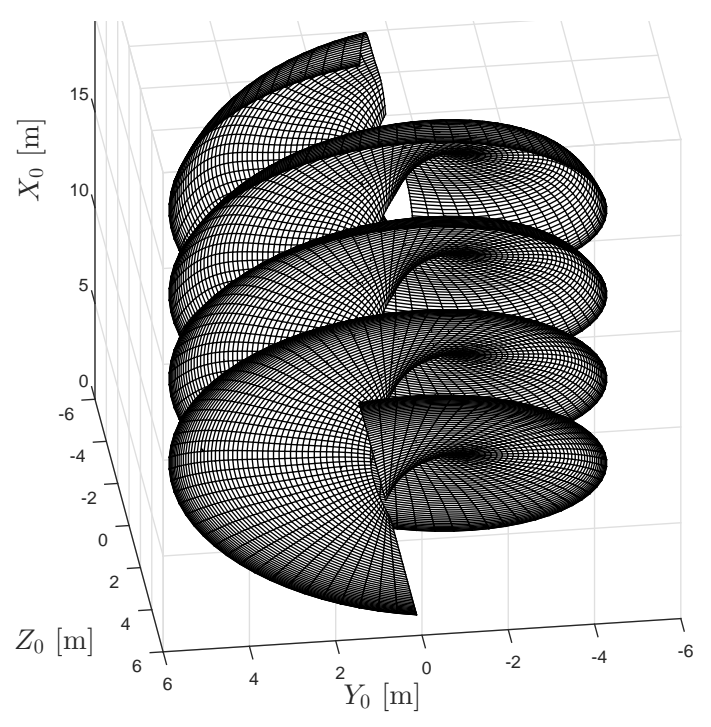

Figure 18. The assumed wake geometry

report by Jonkman [26] are used. Then the circulation at the $i^{\text {th }}$ control point of a blade is given by

$$
\Gamma_{i}=\frac{1}{2} c_{i}\left(U_{\text {resultant }, i}\right) C_{l, i}
$$

where $c_{i}$ is the local chord length and $U_{\text {resultant }, i}$ is the local resultant velocity. After estimating the induced velocity using the Biot Savart Law as outlined before, a new guess to the effective angle of attack at each control point can be derived, followed by new estimates to $C_{l, i}$ and $\Gamma_{i}$. The solution is considered converged when

$$
\left|\Gamma_{i, s+1}-\Gamma_{i, s}\right| \leq 10^{-5}
$$

between iterative steps $s+1$ and $s$ for all the control points.

To validate the current rotor aerodynamic calculation, the spanwise variation of effective angle of attack is computed and compared with the experimental measurement obtained by NREL for this wind turbine in the NASA Ames Research Centre [25]. Four cases with freestream velocity $U_{\infty}$ of 7,10, 13 and $15 \mathrm{~m} / \mathrm{s}$ are studied, and the results are plotted on Figure 19. Note that for each blade, only the part with the S809 aerofoil as the cross-sectional shape (i.e. the outer $83.5 \%$ span of a blade) is modelled. It can be seen that the current results are in good agreement with the corresponding NREL measurements, especially for $U_{\infty}=7 \mathrm{~m} / \mathrm{s}$ and $10 \mathrm{~m} / \mathrm{s}$. For $U_{\infty}=13 \mathrm{~m} / \mathrm{s}$ and $15 \mathrm{~m} / \mathrm{s}$, relatively larger discrepancies to the NREL measurements between $r / b$ of approximately 50 and $90 \%$ can be observed compared to the other two cases. These discrepencies could be due to measurement inaccuracy [26] and the assumptions taken in the current rotor aerodynamic model, which might be improved by better simulating the effects of flow separation, tip vortices, incident freestream velocity profile, blade wake and blade deflections.

The current wind turbine aerodynamic code is then linked to the current BPMM-PVII TE noise model. The case with $U_{\infty}=7 \mathrm{~m} / \mathrm{s}$ is considered since the maximum $\alpha$ is less than $10^{\circ}$ for this case, and is below the S809 stall angle of approximately $20^{\circ}$. Hence only pre-stall noise model formulations, such as Equations 2, 3 and 4 associated with the high-frequency directivity function of TE noise (Equation 5), are used. The location of the observer on the ground level at a distance of one hub-height downstream of the wind turbine is depicted in Figure 20. The computed 1/3-octave SPL spectra from five different spanwise stations for a blade at the azimuth of $135^{\circ}\left(0^{\circ}\right.$ aligned with the global $Z_{0}$ direction) are presented in Figure 21. It can be seen in the figure that low frequency (order of $10 \mathrm{Ho} 100 \mathrm{~Hz}$ ) components 

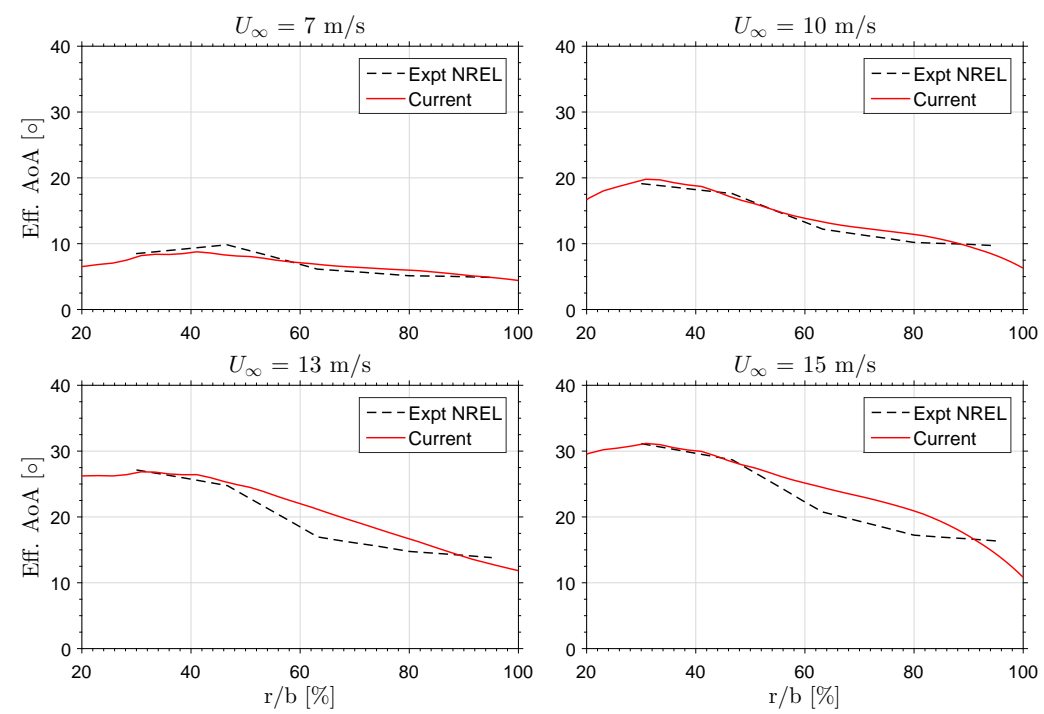

Figure 19. Spanwise effective angle of attack distributions for four different freestream velocities $(y$ is the spanwise location, $b$ is the blade span)

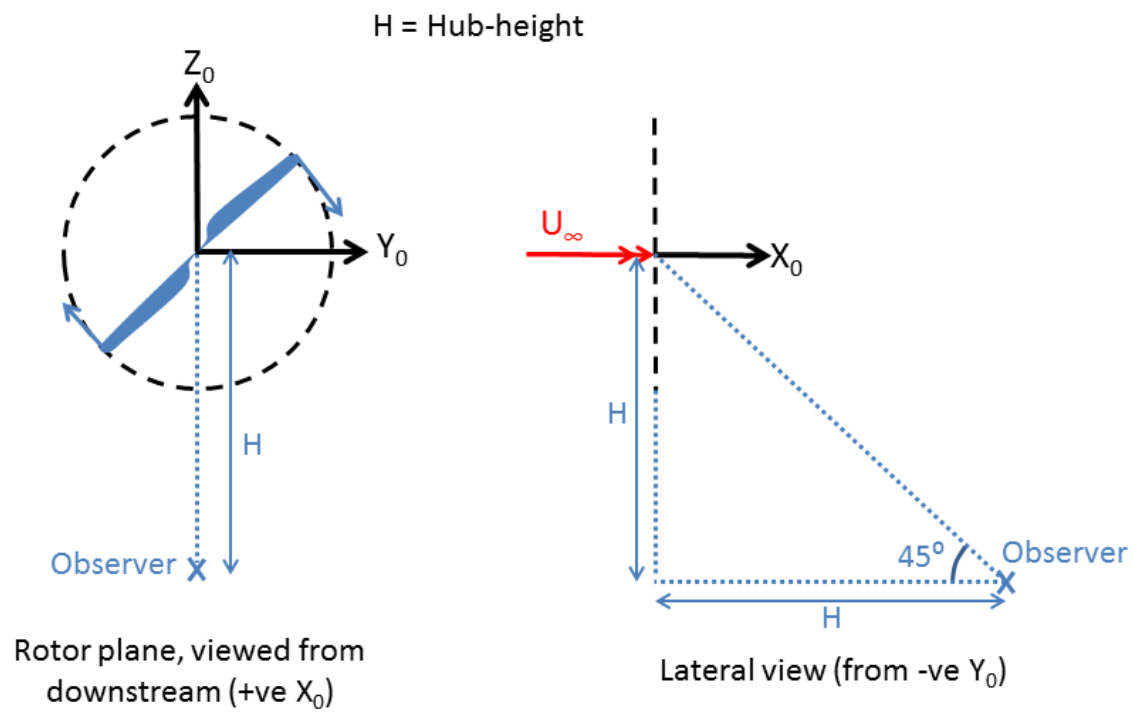

Figure 20. The location of the downwind observer

are delivered mainly from the inboard section of a blade whereas the outboard section is responsible for the higher frequency components. This spanwise variation in the sound spectrum is due to the increasing resultant flow velocity and the decreasing effective angle of attack moving towards the tip. A similar spanwise shift in noise source peak location with frequency has also been observed by Cho et al. [27] in their wind-tunnel experiment with a scaled model of the NREL Phase-VI wind turbine.

The rotor sound-pressure-level contour plots for four different frequencies $(50,100,1000$ and $2000 \mathrm{~Hz})$ predicted at the same observer location are shown in Figure 22. The hub and the blade inboard area which is not based on the S809 aerofoil are excluded in the calculation. The blades rotate clockwise in this view from downstream of the rotor. In addition to confirming that high frequency noise is mainly emitted from the outboard section of the blades, it can also be seen from 


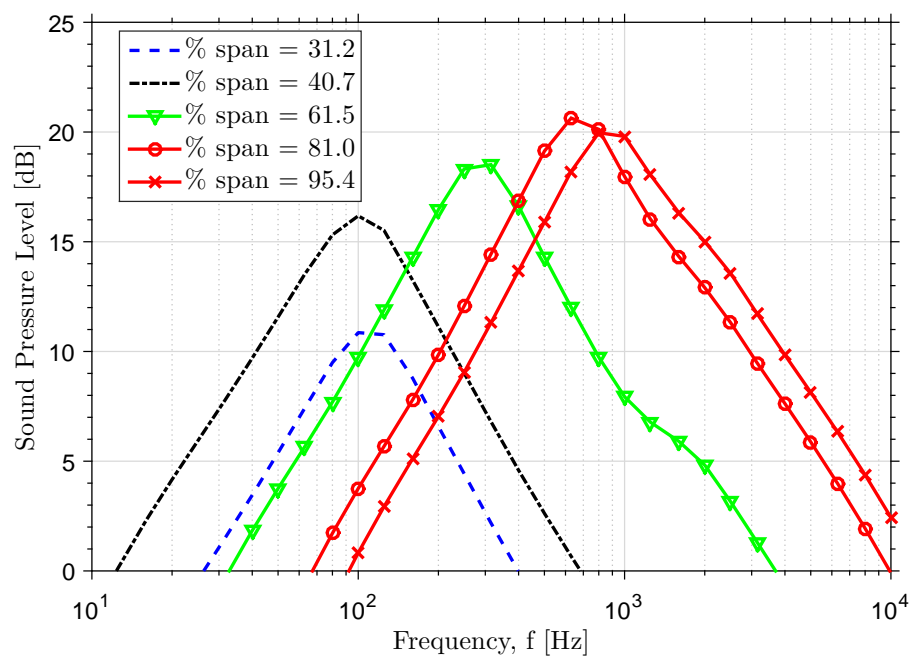

Figure 21. SPL spectra from five different spanwise positions along a blade at the azimuth of $135^{\circ}$ observed at $\left(X_{0}, Y_{0}, Z_{0}\right)=$ (12.192m, 0.0m, -12.192m)

Figure 22 that much of the noise perceived by the observer is due to the downward movement of a blade. This is due to the high-frequency TE noise directivity function used [9], which leads to louder noise radiated towards the local blade section upwind direction. Hence if an observer is located above the wind turbine at a position of $\left(X_{0}, Y_{0}, Z_{0}\right)=(12.192 \mathrm{~m}, 0.0 \mathrm{~m}$, $12.192 \mathrm{~m})$, more noise would be perceived by the upward movement of a blade.
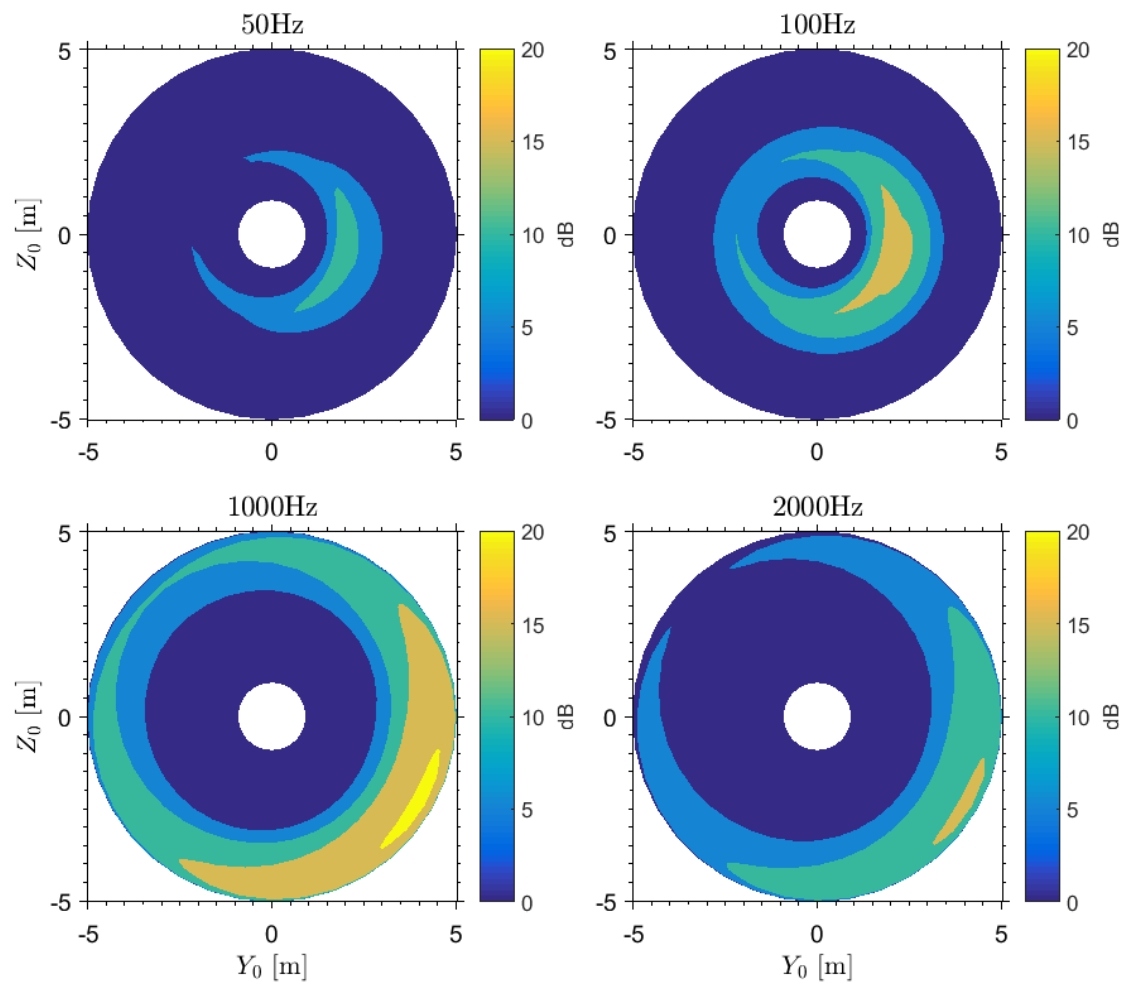

Figure 22. SPL contour plots for four different frequencies radiated from the rotor plane to an observer located at $\left(X_{0}, Y_{0}, Z_{0}\right)=$ (12.192m, 0.0m, -12.192m) 
The current rotor noise estimation methodology which utilises the proposed BPMM-PVII model forms a good basis for further developments which would benefit the wind-turbine industry for their design optimisation process aimed at high aerodynamic performance and low noise. Improvements such as the incorporation of convective amplification and Doppler-shifted frequency will make the current method more relevant to real wind turbine rotor noise estimation.

\section{CONCLUSION}

In the current study, improvements to the semi-empirical BPM TE noise model have been proposed based on the wind-tunnel-measured aeroacoustic data for aerofoils used on modern wind turbine blades. The two current enhanced models are denoted as BPMM-PVII and BPMM-BL $k \omega$, where the former uses a panel method with viscous-inviscid interaction implemented and the latter employs a two-dimensional Reynolds-averaged Navier-Stokes model for boundarylayer calculations. It has been shown that the SPL spectra predicted by both enhanced BPM models are much closer to experimental measurements than those by the original BPM model, even for aerofoils whose measured data have not been used in the derivation of the improved formulations. Both enhanced BPM models also out-perform two recent anisotropic TNO models for many test cases analysed. Among the six TE noise models tested, the predictions by the current BPMMPVII model show the closest agreement to the experimental measurements. The BPMM-PVII model has been integrated with a wind turbine rotor aerodynamic code based on Prandtl's nonlinear lifting line theory. The current aerodynamic code has shown reasonably good agreement with experimental results in the prediction of spanwise effective angle of attack. With some further improvements, the current methodology will be able to facilitate the process of wind-turbine design optimisation aimed at high aerodynamic performance and low noise.

\section{ACKNOWLEDGEMENT}

The authors gratefully acknowledge the support of Knowledge Transfer Secondment (KTS), Engineering and Physical Sciences Research Council (EPSRC, EP/J007633/1) and Vestas Technology R\&D in the completion of this work.

\section{REFERENCES}

1. J. Ladenburg, M. Termansen, and B. Hasler, "Assessing acceptability of two onshore wind power development schemes: a test of viewshed effects and the cumulative effects of wind turbines," Energy, vol. 54, pp. 45-54, 2013.

2. X. Sun, D. Huang, and G. Wu, “The current state of offshore wind energy technology development," Energy, vol. 41, pp. 298-312, 2012.

3. T. Brooks, D. Pope, and M. Marcolini, "Airfoil self-noise and prediction,” tech. rep., NASA, 1989.

4. M. Lowson, "A new prediction model for wind turbine noise," in Renewable Energy Int. Conference on Clean Power, pp. 177-182, 1993.

5. P. Moriarty and P. Migliore, "Semi-empirical aeroacoustic noise prediction code for wind turbines," tech. rep., NREL, 2003.

6. P. Moriarty, G. Guidati, and P. Migliore, "Prediction of turbulent inflow and trailing-edge noise for wind turbines," in 11th AIAA/CEAS Aeroacoustics Conference, 2005.

7. S. Oerlemans, P. Sijtsma, and B. Mendez Lopez, "Location and quantification of noise sources on a wind turbine," $J$. Sound Vib., vol. 299(4-5), pp. 869-883, 2007.

8. S. Oerlemans, M. Fisher, T. Maeder, and K. Kogler, "Reduction of wind turbine noise using optimized airfoils and trailing-edge serrations," in 14th AIAA/CEAS Aeroacoustics Conference, 2008. 
9. S. Oerlemans and J. Schepers, "Prediction of wind turbine noise and validation against experiment," Int. J. Aeroacoustics, vol. 8(6), pp. 555-584, 2009.

10. W. Shen and J. Sorensen, “Aero-acoustic modelling using large eddy simulation,” J. Phys. Conf. Ser., vol. $75,2007$.

11. A. Tadamasa and M. Zangeneh, "Numerical prediction of wind turbine noise," Renew. Energ., vol. 36(7), pp. 19021912, 2011.

12. R. Ewert, J. Dierke, J. Siebert, A. Neifeld, C. Appel, M. Siefert, and O. Kornow, "CAA broadband noise prediction for aeroacoustic design,” J. Sound Vib., vol. 330(17), pp. 4139-4160, 2011.

13. R. Parchen, "Progress report DRAW: a prediction scheme for trailing-edge noise based on detailed boundary-layer characteristics,” Tech. Rep. TNO Rept. HAG-RPT-980023, TNO Institute of Applied Physics, 1998.

14. T. Lutz, A. Herrig, W. Wurz, M. Kamruzzaman, and E. Kramer, "Design and wind-turbine verification of low-noise airfoils for wind turbines," AIAA J., vol. 45(4), pp. 779-785, 2007.

15. F. Bertagnolio, A. Fischer, and W. Zhu, "Tuning of turbulent boundary layer anisotropy for improved surface pressure and trailing-edge noise modeling," J. Sound Vib., vol. 333(3), pp. 991-1010, 2014.

16. M. Kamruzzaman, T. Lutz, W. Wurz, W. Shen, W. Zhu, M. Hansen, F. Bertagnolio, and H. Madsen, "Validations and improvements of airfoil trailing-edge noise prediction models using detailed experimental data," Wind Energy, vol. 15, pp. 45-61, 2012.

17. D. Wilcox, Turbulence modeling for CFD. DCW Industries, Inc., second ed., 2000.

18. M. Drela, Low Reynolds number aerodynamics. Springer Berlin Heidelberg, 1989.

19. A. Herrig, W. Wurz, E. Kramer, and S. Wagner, "New CPV-results of NACA 0012 trailing-edge noise," in International Conference of Methods of Aerophysical Research, 2008.

20. B. Plogmann and W. Wurz, "Aeroacoustic measurements on a NACA 0012 applying the coherent particle velocity method," Exp. Fluids, vol. 54(7), 2013.

21. M. Fink, “Noise components method for airframe noise,” J. Aircraft, vol. 16(10), pp. 659-665, 1977.

22. M. Herr and M. Kamruzzaman, "Benchmarking of trailing-edge noise computations - outcome of the BANC-II Workshop," in 19th AIAA/CEAS Aeroacoustics Conference Proc., 2013.

23. P. Lysak and T. Brungart, "Velocity spectrum model for turbulence ingestion noise from computational-fluiddynamics calculations," AIAA J. Technical Notes, vol. 41(9), pp. 1827-1829, 2003.

24. M. Kamruzzaman, D. Bekiropoulos, A. Wolf, T. Lutz, and E. Kramer, "Rnoise: a RANS based airfoil trailing-edge noise prediction model," in 20th AIAA/CEAS Aeroacoustics Conference Proc., 2014.

25. M. Hand, D. Simms, L. Fingersh, D. Jager, J. Cotrell, S. Schreck, and S. Larwood, "Unsteady aerodynamics experiment phase VI: wind tunnel test configurations and available data campaigns,” tech. rep., NREL, 2001.

26. J. Jonkman, "Modeling of the UAE wind turbine for refinement of FAST_AD," tech. rep., NREL, 2003.

27. T. Cho, C. Kim, and D. Lee, "Acoustic measurement for $12 \%$ scaled model of NREL Phase VI wind turbine by using beamforming," Curr. Appl. Phys., vol. 10(2), Supplement, pp. S320-S325, 2010. 